\title{
Whittaker Functions for the Large Discrete Series Representations of $S U(2,1)$ and Related Zeta Integrals
}

\author{
Dedicated to Professor Hideo Shimizu on his 60th birthday
}

By

\author{
Harutaka KoseKI ${ }^{*}$ and Takayuki ODA ${ }^{* *}$
}

\begin{abstract}
In terms of classical Bessel function, we represent explicitly the radial part of Whittaker functions on $S U(2,1)$ belonging to the large discrete series representations. Moreover we compute archimedean local $L$-factors corresponding to a construction of $L$-function by Gelbart and Piatetski-Shapiro.
\end{abstract}

\section{§ 0. Introduction}

The real semisimple group $S U(2,1)$ has three types of discrete series: one is holomorphic discrete series, another anti-holomorphic, and the other neither holomorphic nor anti-holomorphic. The representations of the last type are large, in the sense of Vogan [V], namely their Gel'fand-Kirillov dimensions coincide with the dimension of the maximal unipotent subgroup. A theorem of Kostant $[\mathrm{K}]$ then assures, for such a representation, the existence (and the uniqueness) of the Whittaker model in the $C^{\infty}$-context. In this paper, we give an explicit formula for (the Mellin transforms of) $K$-finite vectors in such a Whittaker model, and applying this formula we evaluate certain local zeta integrals.

Firstly, we express the minimal $K$-type Whittaker functions in terms of the modified Bessel functions (Theorem 4.5). The computation is based on the method of Yamashita [Y-I], [Y-II]. With this result in hand we calculate the Mellin transforms of general $K$-finite Whittaker functions (Theorem 5.5). Here we use the fact that the representation is $K$-multiplicity free.

Communicated by Y. Ihara, July 14, 1994.

1991 Mathematics Subject Classification(s): 11F70, 43A90

* Department of Mathematics, Fac. of Education, Mie University, Tsu, Mie Pref. , 514 Japan.

** Department of Mathematical Science, The University of Tokyo, Komaba, Tokyo, 153 Japan. 
To a generic representation of the quasi-split unitary group in three variables over a local field, Gelbart and Piatetski-Shapiro [G-Ps] attached certain zeta integrals. Among other things, they computed explicitly such integrals for unramified Whittaker functions in the case where the base field is $p$-adic. They turn out to be Langlands' local $L$-factors in this case.

We shall compute the same local zeta integrals in the case where the base field is real and the representation belongs to the large discrete series of $U(2,1)$. We then express the "greatest common divisor" as a product of three gamma functions (Theorem 6.8). A local zeta integral depends on the choices of a $K$-finite vector in the Whittaker model of a given representation of $U(2,1)$, a quasi-character of $\mathbf{C}^{\times}$, and a $M$-finite test function in the Schwartz-Bruhat functions on $\mathbf{C}^{2}$. Here $M$ is the toral part of the Levi component of the standard minimal parabolic subgroup of $S U(2,1)$. The zeta integral vanishes if the $M$-types of these three choices are not compatible. Therefore it is necessary to consider all the $K$-types to find a test function of good $M$-type.

\section{Contents}

$\S 1$. Root Space Decomposition and Iwasawa Decomposition

1.1. Basic notation

1.2. Root system and root space decomposition

1.3. Iwasawa decomposition of root vectors

$\S 2$. Irreducible $K$-modules and their Tensor Products with $\Downarrow_{\mathbf{c}}$

2.1. Parametrization of irreducible $K$-modules

2.2. Tensor products with $\mathfrak{H}_{\mathrm{c}}$

$\S 3$. Whittaker Models of Discrete Series and Schmid Operator

3.1. Parametrization of the discrete series representations and their $K$-types

3.2. Whittaker functionals

3.3. Differential operator $\mathscr{D}_{\eta, \mu}$

$\S 4$. Explicit Formula for the Minimal $K$-type Whittaker Functions

4.1. Radial part of $\nabla_{\eta, \mu}^{ \pm}$and their projections

4.2. The minimal $K$-type Whittaker functions

§ 5. An Explicit Formula for $K$-finite Whittaker Functions

5.1. Recurrence relations for $K$-finite vectors in $W h_{\eta}\left(\pi_{\lambda}^{*}\right)$

5.2. The Mellin transforms of $K$-finite Whittaker functions

5.3. The case of $U(2,1)$

§ 6. The Local Zeta Integrals and the Gamma Factor

6.1. The Mellin transforms $F_{\Phi}^{\omega}(h ; s)$

6.2. The local zeta integrals $L^{\omega}(s, \tilde{W}, \Phi)$ and their greatest common divisor

6.3. Proof of Theorem (6.8)

$\S 7$. Comments for the Case of the Principal Series Representations

$\S 8$. Comments on $L$-function and its Special Values 


\section{§ 1. Root Space Decomposition and Iwasawa Decomposition}

In this section, we fix basic notation recalling the fundamental results on the structure of $S U(2,1)$ and the associated Lie algebra $\mathfrak{s u}(2,1)$.

\subsection{Basic notation}

Let $G L(3, \mathbf{C})$ and $S L(3, \mathbf{C})$ be the complex general linear group and special linear group of degree 3 , respectively. We denote by $\operatorname{diag}\left(x_{1}, x_{2}, x_{3}\right)$ a diagonal matrix of degree 3 with $(i, i)$-entry $x_{\imath}$ for each $i=1,2$, 3. Put $I_{2,1}=\operatorname{diag}(1,1,-1)$. Then we denote by $G$ the group $S U(2,1)$ defined by

$$
S U(2,1)=\left\{g \in S L(3, \mathbf{C}) \mid{ }^{t} \bar{g} I_{2,1} g=I_{2,1}\right\}
$$

Here ${ }^{t} g$ is the transpose of $g$, and $\bar{g}$ the complex conjugate of $g$.

We fix a maximal compact subgroup $K$ of $G$ once for all:

$K=\left\{\left(\begin{array}{lll}g_{11} & g_{12} & 0 \\ g_{21} & g_{22} & 0 \\ 0 & 0 & g_{33}\end{array}\right) \in G \mid\left(\begin{array}{ll}g_{11} & g_{12} \\ g_{21} & g_{22}\end{array}\right) \in U(2), g_{33} \in U(1), \operatorname{det}\left(\begin{array}{ll}g_{11} & g_{12} \\ g_{21} & g_{22}\end{array}\right) \cdot g_{33}=1\right\}$.

The homogeneous space $G / K$ is a Hermitian symmetric domain, isomorphic to a complex hyperball of dimension 2 .

\subsection{Root system and root space decomposition}

The Lie algebra $g$ of $G$ is given by

$$
\begin{aligned}
\mathfrak{5 H}(2,1)= & \left\{X \in M_{3}(\mathbf{C}) \mid{ }^{t} \bar{X} \cdot I_{2,1}+I_{2,1} X=0\right\} \\
& =\left\{\left(\begin{array}{ll}
X_{1} & X_{2} \\
{ }^{t} \bar{X}_{2} & X_{3}
\end{array}\right) \mid \begin{array}{ll}
X_{1} \in \mathfrak{U}(2), & X_{2} \in M_{2,1}(\mathbf{C}), \quad X_{3} \in \mathfrak{U}(1), \\
\operatorname{tr} X_{1}+X_{3}=0
\end{array}\right\} .
\end{aligned}
$$

The Lie algebra of $K$ is

$$
\mathfrak{l}=\left\{\left(\begin{array}{ll}
X_{1} & 0 \\
0 & X_{3}
\end{array}\right) \mid X_{1} \in \mathfrak{u}(2), X_{3} \in \mathfrak{u}(1), \operatorname{tr} X_{1}+X_{3}=0\right\} .
$$

Put

$$
\mathfrak{p}=\left\{\left(\begin{array}{ll}
0 & X_{2} \\
t \bar{X}_{2} & 0
\end{array}\right) \mid X_{2} \in M_{2,1}(\mathbf{C})\right\} .
$$

Then we have a Cartan decomposition 


$$
\mathfrak{g}=\mathfrak{l} \oplus \mathfrak{l} .
$$

Since $G / K$ is hermitian, we have a decomposition

$$
\mathfrak{g}_{\mathbf{c}}=\mathfrak{f}_{\mathbf{c}} \oplus \mathfrak{p}_{+} \oplus \mathfrak{h}_{-},
$$

such that $\mathfrak{h}_{+}$is identified with the holomorphic tangent space at the origin $1 \cdot K \in G / K$.

We have

$$
\mathfrak{p}_{+}=\left\{\left(\begin{array}{l|l}
0 & X_{2} \\
\hline 0 & 0
\end{array}\right) \mid X_{2} \in M_{2,1}(\mathbf{C})\right\}
$$

and

$$
\mathfrak{p}_{-}=\left\{\left(\begin{array}{l|l}
0 & 0 \\
\hline{ }^{t} Y_{2} & 0
\end{array}\right) \mid Y_{2} \in M_{2,1}(\mathbf{C})\right\} .
$$

We fix a compact Cartan subalgebra $t$ in by

$$
\mathrm{t}=\left\{\operatorname{diag}\left(\sqrt{-1} h_{1}, \sqrt{-1} h_{2}, \sqrt{-1} h_{3}\right) \mid h_{\imath} \in \mathbf{R}, \sum_{\imath=1}^{3} h_{\imath}=0\right\}
$$

The root system $\Sigma=\Sigma\left(g_{c}, t_{c}\right)$ is given as follows. We define linear forms $\beta_{i j}$ on $t_{c}$ by

$$
\beta_{i j}\left(\operatorname{diag}\left(h_{1}, h_{2}, h_{3}\right)\right)=h_{i}-h_{j}(i \neq j, 1 \leq i, j \leq 3) .
$$

Then $\Sigma=\left\{\beta_{i j} \mid i \neq j, 1 \leq i, j \leq 3\right\}$. We fix a positive root system $\Sigma_{+}=$ $\left\{\beta_{i j} \mid i<j\right\}$.

Let $\mathrm{g}_{\beta}$ be the root space associated to $\beta \in \Sigma$. Then $\mathrm{g}_{\beta} \subset \mathfrak{l}_{\mathrm{C}}$ if $\beta \in\left\{\beta_{12}, \beta_{21}\right\}$, and $\mathrm{g}_{\beta} \subset \mathfrak{l}_{\mathrm{c}}$ if $\beta \notin\left\{\beta_{12}, \beta_{21}\right\}$. The set of compact roots is given by $\Sigma_{C}=\left\{\beta_{12}, \beta_{21}\right\}$, and the set of non-compact roots by

$$
\Sigma_{n}=\Sigma-\Sigma_{c}=\left\{\beta_{13}, \beta_{23}, \beta_{31}, \beta_{32}\right\}
$$

Put $\Sigma_{c,+}=\Sigma_{c} \cap \Sigma_{+}$and $\Sigma_{n,-}=\Sigma_{n} \cap \Sigma_{+}$.

Let $E_{\imath j}$ be a matrix of degree 3 with $(k, l)$ entries $\delta_{k \imath} \delta_{l j}\left(\delta_{k \imath}\right.$ are Kronecker delta). Then $E_{i}$, is a generator of the root space $g_{\beta_{\imath}}$. We define $X_{\beta_{\imath}}$, by

$$
X_{\beta_{\imath \jmath}}= \begin{cases}E_{i j} & ((i, j) \neq(2,1)) \\ -E_{\imath \jmath} & ((i, j)=(2,1)) .\end{cases}
$$




\subsection{Iwasawa decomposition}

A maximal abelian subalgebra $\mathfrak{a}$ of $\mathfrak{p}$ is given by $\mathfrak{a}=\mathbf{R} \cdot H_{1}$ with

$$
H_{1}=\left(\begin{array}{lll}
0 & 0 & 1 \\
0 & 0 & 0 \\
1 & 0 & 0
\end{array}\right)
$$

We define a linear form $e_{1}$ on $\mathfrak{a}$ by $e_{1}\left(H_{1}\right)=1$. The root space $\mathfrak{g}_{2 e_{1}}$ is $\mathbf{R} E_{1}$ with

$$
E_{1}=i\left(\begin{array}{ccc}
1 & & -1 \\
& 0 & \\
1 & & -1
\end{array}\right)
$$

From now on we use the convention that unwritten components of a matrix are zero. Put

$$
E_{2,+}=\left(\begin{array}{ccc} 
& -1 & \\
1 & & -1 \\
& -1 &
\end{array}\right), E_{2,-}=\left(\begin{array}{ccc} 
& -i \\
-i & & i \\
& -i
\end{array}\right)
$$

Then the root space $g_{e_{1}}$ is $\mathbf{R} E_{2,+} \oplus \mathbf{R} E_{2,-}$. Set

$$
\mathfrak{n}_{2 e_{1}} \text { is } \mathfrak{g}_{e_{1}} \oplus \mathfrak{g}_{2 e_{1}}=\mathbf{R} E_{1} \oplus \mathbf{R} E_{2,+} \oplus \mathbf{R} E_{2,-} \text {. }
$$

Then $\mathfrak{n}$ is a maximal nilpotent subalgebra of $\mathfrak{g}$. We define elements $H_{12}^{\prime}, H_{13}^{\prime}$, and $Z$ of $t_{c}$ by

$$
\begin{gathered}
H_{12}^{\prime}=\left(\begin{array}{ccc}
1 & & \\
& -1 & \\
& & 0
\end{array}\right), H_{13}^{\prime}=\left(\begin{array}{ccc}
1 & & \\
& 0 & \\
& & -1
\end{array}\right), \\
\quad \text { and } Z=2 H_{13}^{\prime}-H_{12}^{\prime}=\left(\begin{array}{lll}
1 & & \\
& 1 & \\
& & -2
\end{array}\right) .
\end{gathered}
$$

Here is the Iwasawa decomposition $\mathfrak{g}_{\mathbf{c}}=\mathfrak{l}_{\mathbf{c}} \oplus \mathfrak{a}_{\mathbf{c}} \oplus \mathfrak{n}_{\mathbf{c}}$ of the root vectors $X_{\beta_{\imath}}$ associated to non-compact roots. 
Lemma (1.1).

$$
\begin{aligned}
& X_{13}=\frac{1}{2} H_{13}^{\prime}+\frac{1}{2} H_{1}+\frac{i}{2} E_{1} ; \\
& X_{31}=-\frac{1}{2} H_{13}^{\prime}+\frac{1}{2} H_{1}-\frac{i}{2} E_{1} ; \\
& X_{23}=-X_{21}-\frac{1}{2} E_{2,+}-\frac{i}{2} E_{2,-} ; \\
& X_{32}=-X_{12}-\frac{1}{2} E_{2,+}+\frac{i}{2} E_{2,-} .
\end{aligned}
$$

\section{$\S 2$. Irreducible $K$-modules and their Tensor Products with $\mathfrak{l}_{\mathrm{c}}$}

In this section, we firstly parametrize integral linear forms on $t_{c}$ and irreducible $K$-modules. We then describe, for an arbitrary irreducible $K$-module $V$, the projectors from the $K$-module $V \otimes \mathfrak{P}_{\mathrm{c}}$ onto irreducible components in terms of "standard basis". We later apply this explicit description to the study of differential equations satisfied by Whittaker functions.

\subsection{Parametrization of irreducible $K$-modules}

We fix a Cartan subgroup $T$ of $K$, which is also a compact Cartan subgroup of $G$, by

$$
T:=\exp (\mathrm{t})=\left\{\left(\begin{array}{lll}
e^{i \alpha} & & \\
& e^{i \beta} & \\
& & e^{-i(\alpha+\beta)}
\end{array}\right) \mid \alpha, \beta \in \mathbf{R}\right\} .
$$

We denote by $\hat{T}$ and $L_{T}$, respectively, the group of unitary characters of $T$ and the lattice of $T$-integral forms. Then $L_{T}$ is a lattice in $\sqrt{-1} t^{*}, \mathrm{t}^{*}=\operatorname{Hom}_{\mathbf{R}}(\mathrm{t}, \mathbf{R})$, and there exists a canonical isomorphism

$$
\hat{T} \cong L_{T}, \chi \mapsto \text { the differential of } \chi
$$

In this paper we identify $L_{T}$ with $\mathbf{Z}^{\oplus 2}$ by the following

Convention (2.1). The differential of a unitary character

$$
T \ni\left(\begin{array}{lll}
e^{i \alpha} & & \\
& e^{i \beta} & \\
& & e^{-i(\alpha+\beta)}
\end{array}\right) \mapsto e^{i(m \alpha+n \beta)} \in \mathbf{C}^{(1)}
$$

is identified with $(m, n) \in \mathbf{Z}^{\oplus 2}$. ( $\mathbf{C}^{(1)}$ stands for the group of complex numbers 
with absolute value one.)

The roots $\beta_{i} \in \Sigma=\Sigma\left(g_{\mathrm{c}}, \mathrm{t}_{\mathrm{c}}\right)$ are $T$-integral, and are parametrized as follows: compact roots: $\beta_{12}=(1,-1), \beta_{21}=-\beta_{12}=(-1,1)$

non-compact roots: $\beta_{13}=(2,1), \beta_{31}=-\beta_{13}=(-2,-1)$,

$$
\beta_{23}=(1,2), \beta_{32}=-\beta_{23}=(-1,-2) \text {. }
$$

The inner product on $L_{T}$ induced from the Killing form is given, up to constant multiple, by

$$
<(m, n),\left(m^{\prime}, n^{\prime}\right)>=2 m m^{\prime}+2 n n^{\prime}-m n^{\prime}-n m^{\prime} .
$$

Thus, the set $L_{T}^{+}$of dominant $T$-integral forms (dominant with respect to the system $\Sigma_{c,+}=\left\{\beta_{12}\right\}$ of compact positive roots) is given by $L_{T}^{+}=\{(m, n)$ $\left.\in \mathbf{Z}^{\oplus 2} \mid m \geq n\right\}$.

The irreducible $K$-modules are parametrized by the above set via highest weight theory. For $\mu=\left(\mu_{1}, \mu_{2}\right) \in L_{T}^{+}$, we denote by $\left(\tau_{\mu}, V_{\mu}\right)$ the irreducible representation of $K \simeq U(2)$ with highest weight $\mu$. The associated irreducible representations of $K_{\mathbf{C}} \simeq G L(2, \mathbf{C})$ and $\mathfrak{l}_{\mathbf{C}} \simeq \mathfrak{g}_{1}(2, \mathbf{C})$ will be denoted by the same symbol. The degree of $\left(\tau_{\mu}, V_{\mu}\right)$ is given by $\operatorname{dim}_{\mathrm{C}} V_{\mu}=d_{\mu}+1, d_{\mu}:=\mu_{1}-\mu_{2}$. The restriction of $\tau_{\mu}$ to $T$ splits into the following $T$-weights: $\mu$ (=the highest weight), $\mu-\beta_{12}, \cdots, \mu-d_{\mu} \beta_{12}$ (= the lowest weight). We denote by $\left(\tau_{\mu}^{*}, V_{\mu}^{*}\right)$ the contragredient of $\left(\tau_{\mu}, V_{\mu}\right)$. Then $\nu \in L_{T}^{+}$appears in $\left(\tau_{\mu}^{*}, V_{\mu}^{*}\right)$ as a $T$-weight if and only if $-\nu$ appears in $\left(\tau_{\mu}, V_{\mu}\right)$. Hence

$$
\left(\tau_{\mu}^{*}, V_{\mu}^{*}\right)=\left(\tau_{\mu}^{*}, V_{\mu}^{*}\right), \quad \mu^{*}:=\text { the highest weight of }\left(\tau_{\mu}^{*}, V_{\mu}^{*}\right)=-\mu+d_{\mu} \beta_{12}
$$

Each irreducible $\mathfrak{f}_{\mathrm{C}}$-module $\left(\tau_{\mu}, V_{\mu}\right)$ has a basis $\left\{v_{k}^{\mu} \mid 0 \leq k \leq d_{\mu}\right\}$ on which the elements $Z, H_{12}^{\prime}, H_{13}^{\prime}, X_{\beta_{12}}, X_{\beta_{21}}$ of $\mathfrak{l}_{\mathrm{c}}$ act as follows:

$$
\begin{aligned}
\tau_{\mu}(Z) v_{k}^{\mu} & =\left(\mu_{1}+\mu_{2}\right) v_{k}^{\mu}, \\
\tau_{\mu}\left(H_{12}^{\prime}\right) v_{k}^{\mu} & =\left\{\mu-\left(d_{\mu}-k\right) \beta_{12}\right\}\left(H_{12}^{\prime}\right) v_{k}^{\mu}=\left(2 k-d_{\mu}\right) v_{k}^{\mu}, \\
\tau_{\mu}\left(H_{13}^{\prime}\right) v_{k}^{\mu} & =\left\{\mu-\left(d_{\mu}-k\right) \beta_{12}\right\}\left(H_{13}^{\prime}\right) v_{k}^{\mu}=\left(k+\mu_{2}\right) v_{k}^{\mu}, \\
\tau_{\mu}\left(X_{\beta_{12}}\right) v_{k}^{\mu} & =(k+1) v_{k+1}^{\mu}, \\
\tau_{\mu}\left(X_{\beta_{21}}\right) v_{k}^{\mu} & =\left(k-d_{\mu}-1\right) v_{k-1}^{\mu} .
\end{aligned}
$$

Hence $v_{d_{\mu}}^{\mu}$ and $v_{0}^{\mu}$ are a highest weight vector and a lowest weight vector, respectively. This basis $\left\{v_{k}^{\mu} \mid 0 \leq k \leq d_{\mu}\right\}$ is unique up to multiplication by a common non-zero scalar. We fix $\left\{v_{k}^{\mu} \mid 0 \leq k \leq d_{\mu}\right\}$ for each $\mu$, and call it the standard basis of $V_{\mu}$. 
Throughout this paper we shall keep the following

Convention (2.2). Suppose given a $K$-module $M$, isomorphic to $V_{\mu}$ or $V_{\mu}^{*}$. Let $\left\{f_{k} \mid 0 \leq k \leq d_{\mu}\right\}$ and $\left\{g_{k} \mid 0 \leq k \leq d_{\mu}\right\}$ be two families of elements of $M$. We then use the notation $\sim$ in the following way:

$$
\begin{aligned}
f_{k} \sim g_{k} \Leftrightarrow & \text { there exists a constant } \gamma \in \mathbf{C}^{\times}, \text {independent of } k, \\
& \text { such that } f_{k}=\gamma g_{k} \text { for } k=0, \cdots, d_{\mu} .
\end{aligned}
$$

\subsection{Tensor products with $\mathfrak{c}_{\mathrm{c}}$}

We regard the 4-dimensional vector space $\mathfrak{l}_{\mathrm{c}}$ as a $K_{\mathrm{c}^{-}}$or $\mathfrak{L}_{\mathrm{c}^{-}}$-module via the adjoint representation Ad or ad, respectively. Then $\mathfrak{l}_{+}$and $\mathfrak{p}_{-}$are invariant subspaces, and

$$
\begin{aligned}
& \mathfrak{p}_{+}=\mathbf{C} X_{\beta_{13}} \oplus \mathbf{C} X_{\beta_{23}} \simeq V_{\beta_{13}} ; \quad X_{\beta_{13}} \mapsto v_{1}^{\beta_{13}}, \quad X_{\beta_{23}} \mapsto v_{0}^{\beta_{13}}, \\
& \mathfrak{p}_{-}=\mathbf{C} X_{\beta_{32}} \oplus \mathbf{C} X_{\beta_{31}} \simeq V_{\beta_{32}} ; \quad X_{\beta_{32}} \mapsto-v_{1}^{\beta_{32}}, \quad X_{\beta_{31}} \mapsto v_{0}^{\beta_{32}} .
\end{aligned}
$$

Given an irreducible $K$-module $V_{\mu}$ we have $V_{\mu} \otimes \mathfrak{p}_{\mathrm{c}}=\left(V_{\mu} \otimes \mathfrak{p}_{+}\right) \oplus\left(V_{\mu} \otimes \mathfrak{p}_{-}\right)$, and Clebsch-Gordan's theorem implies the following decomposition of $V_{\mu} \otimes \mathfrak{p}_{ \pm}$:

$$
\begin{aligned}
& V_{\mu} \otimes \mathfrak{H}_{+} \simeq V_{\mu+\beta_{13}} \oplus V_{\mu+\beta_{23}} \simeq V_{\mu+\beta_{13}} \oplus V_{\mu-\beta_{32}}, \\
& V_{\mu} \otimes \mathfrak{H}_{-} \simeq V_{\mu+\beta_{32}} \oplus V_{\mu+\beta_{31}} \simeq V_{\mu+\beta_{32}} \oplus V_{\mu-\beta_{13}} .
\end{aligned}
$$

Here we use the convention that $V_{\nu}=(0)$ if $\nu \in L_{T}$ is not dominant. We hence have

$$
V_{\mu} \otimes \mathfrak{P}_{\mathbf{c}} \simeq V_{\mu}^{+} \oplus V_{\mu}^{-}
$$

with

$$
V_{\mu}^{+}:=V_{\mu+\beta_{13}} \oplus V_{\mu+\beta_{32}}, \quad V_{\mu}^{-}:=V_{\mu-\beta_{13}} \oplus V_{\mu-\beta_{32}},
$$

under the above convention.

The above decompositions of $V_{\mu} \otimes \mathfrak{P}_{\mathrm{c}}$ induce the following projectors:

$$
\begin{array}{ll}
P_{1}^{+}(\mu): V_{\mu} \otimes \mathfrak{P}_{\mathrm{c}} \rightarrow V_{\mu+\beta_{13}}, & P_{2}^{+}(\mu): V_{\mu} \otimes \mathfrak{P}_{\mathrm{c}} \rightarrow V_{\mu-\beta_{32}}, \\
P_{1}^{-}(\mu): V_{\mu} \otimes \mathfrak{P}_{\mathrm{c}} \rightarrow V_{\mu+\beta_{32}}, & P_{2}^{-}(\mu): V_{\mu} \otimes \mathfrak{P}_{\mathrm{c}} \rightarrow V_{\mu-\beta_{13}},
\end{array}
$$

and

$$
\begin{aligned}
& P_{1}(\mu):=P_{1}^{+}(\mu) \oplus P_{1}^{-}(\mu): V_{\mu} \otimes \mathfrak{P}_{\mathrm{c}} \rightarrow V_{\mu}^{+}, \\
& P_{2}(\mu):=P_{2}^{+}(\mu) \oplus P_{2}^{-}(\mu): V_{\mu} \otimes W_{\mathrm{c}} \rightarrow V_{\mu}^{-} .
\end{aligned}
$$


In terms of the standard basis, they are expressed as follows:

Proposition (2.3). Under convention (2.2), one has

(1) $P_{1}^{+}(\mu)\left(v_{k}^{\mu} \otimes X_{\beta_{13}}\right) \sim(k+1) v_{k+1}^{\mu+\beta_{13}}$ and $P_{1}^{+}(\mu)\left(v_{k}^{\mu} \otimes X_{\beta_{23}}\right) \sim\left(d_{\mu}-k+1\right) v_{k}^{\mu+\beta_{13}}$ for $0 \leq k \leq d_{\mu}$, and $\left.P_{1}^{+}(\mu)\right|_{V_{\mu} \otimes p_{-}}$is the zero-map,

$\left(1^{\prime}\right) P_{1}^{-}(\mu)\left(v_{k}^{\mu} \otimes X_{\beta_{32}}\right) \sim-(k+1) v_{k+1}^{\mu+\beta_{32}}$ and $P_{1}^{-}(\mu)\left(v_{k}^{\mu} \otimes X_{\beta_{31}}\right) \sim\left(d_{\mu}-k+1\right) v_{k}^{\mu+\beta_{32}}$ for $0 \leq k \leq d_{\mu}$, and $\left.P_{1}^{-}(\mu)\right|_{V_{\mu} \otimes \mathfrak{p}_{+}}$is the zero-map,

(2) $P_{2}^{+}(\mu)\left(v_{k}^{\mu} \otimes X_{\beta_{13}}\right) \sim-v_{k}^{\mu-\beta_{32}}$ and $P_{2}^{+}(\mu)\left(v_{k}^{\mu} \otimes X_{\beta_{23}}\right) \sim v_{k-1}^{\mu-\beta_{32}}$ for $0 \leq k \leq d_{\mu}$ (where we put $v_{k}^{\mu-\beta_{32}}=0$ for $\left.k=-1, d_{\mu}\right)$, and $\left.P_{2}^{+}(\mu)\right|_{V_{\mu} \otimes \mu_{-}}$is the zero-map,

$\left(2^{\prime}\right) P_{2}^{-}(\mu)\left(v_{k}^{\mu} \otimes X_{\beta_{32}}\right) \sim v_{k}^{\mu-\beta_{13}}$ and $P_{2}^{-}(\mu)\left(v_{k}^{\mu} \otimes X_{\beta_{31}}\right) \sim v_{k-1}^{\mu-\beta_{13}}$ for $0 \leq k \leq d_{\mu}$ (where we put $v_{k}^{\mu-\beta_{13}}=0$ for $\left.k=-1, d_{\mu}\right)$, and $\left.P_{2}^{-}(\mu)\right|_{V_{\mu} \otimes \mathfrak{p}_{+}}$is the zero-map.

Here one should note that $d_{\mu \pm \beta_{13}}=d_{\mu \pm \beta_{32}}=d_{\mu} \pm 1$.

Proof. We prove only (1) and (2). The decomposition of $V_{\mu} \otimes \mathfrak{P}_{-}$shows that $\left.P_{j}^{+}(\mu)\right|_{V_{\mu} \otimes \mathcal{H}_{-}}$is the zero-map for $j=1$, 2. In the decomposition

$$
V_{\mu} \otimes \mathfrak{H}_{+}=V_{\mu} \otimes\left(\mathbf{C} X_{\beta_{13}} \oplus \mathbf{C} X_{\beta_{23}}\right) \simeq V_{\mu+\beta_{13}} \oplus V_{\mu-\beta_{32}}
$$

$v_{0}^{\mu} \otimes X_{\beta_{23}}$ is a lowest weight vector of the $V_{\mu+\beta_{13}}$-component. Hence we may put

$$
P_{1}^{+}(\mu)\left(v_{0}^{\mu} \otimes X_{\beta_{23}}\right)=\gamma\left(d_{\mu}+1\right) v_{0}^{\mu+\beta_{13},} \quad P_{2}^{+}(\mu)\left(v_{0}^{\mu} \otimes X_{\beta_{23}}\right)=0
$$

with $\gamma \in \mathbf{C}^{\times}$. The action of the powers of $X_{\beta_{12}}$ on both sides yields

$$
\begin{gathered}
P_{1}^{+}(\mu)\left(v_{k-1}^{\mu} \otimes X_{\beta_{13}}+v_{k}^{\mu} \otimes X_{\beta_{23}}\right)=\gamma\left(d_{\mu}+1\right) v_{k}^{\mu+\beta_{13},} \\
P_{2}^{+}(\mu)\left(v_{k-1}^{\mu} \otimes X_{\beta_{13}}+v_{k}^{\mu} \otimes X_{\beta_{23}}\right)=0,
\end{gathered}
$$

for $0 \leq k \leq d_{\mu}+1$, under the convention $v_{-1}^{\mu}=v_{d_{\mu}+1}^{\mu}=0$. On the other hand, a lowest weight vector of the $V_{\mu-\beta_{32}}$-component is a linear combination of $v_{0}^{\mu} \otimes X_{\beta_{13}}$ and $v_{1}^{\mu} \otimes X_{\beta_{23}}$, and it is annihilated by $X_{\beta_{21}}$. From these facts we find that $-d_{\mu} v_{0}^{\mu}$ $\otimes X_{\beta_{13}}+v_{1}^{\mu} \otimes X_{\beta_{23}}$ is a lowest weight vector, and we get

$$
P_{1}^{+}(\mu)\left(-d_{\mu} v_{0}^{\mu} \otimes X_{\beta_{13}}+v_{1}^{\mu} \otimes X_{\beta_{23}}\right)=0, P_{2}^{+}(\mu)\left(-d_{\mu} v_{0}^{\mu} \otimes X_{\beta_{13}}+v_{1}^{\mu} \otimes X_{\beta_{23}}\right)=\delta v_{0}^{\mu-\beta_{32}}
$$

with $\delta \in \mathbf{C}^{\times}$. The action of the powers of $X_{\beta_{12}}$ on both sides yields

$$
\begin{gathered}
P_{1}^{+}(\mu)\left(\left(k-1-d_{\mu}\right) v_{k-1}^{\mu} \otimes X_{\beta_{13}}+k v_{k}^{\mu} \otimes X_{\beta_{23}}\right)=0, \\
P_{2}^{+}(\mu)\left(\left(k-1-d_{\mu}\right) v_{k-1}^{\mu} \otimes X_{\beta_{13}}+k v_{k}^{\mu} \otimes X_{\beta_{23}}\right)=\delta v_{k-1}^{\mu-\beta_{32}},
\end{gathered}
$$


for $0 \leq k \leq d_{\mu}+1$. The assertion (1) (resp. (2)) is then deduced from (a) and (c) (resp. (b) and (d)).

\section{$\S 3$. Whittaker Model of Discrete Series and Schmid Operator}

\subsection{Parametrization of the discrete series representations and their $K$-types}

Let us recall the Harish-Chandra parametrization of the discrete series representations of $S U(2,1)$.

The Weyl group of $G$ is the symmetric group $\mathfrak{S}_{3}$ of order 3 . Let $\Sigma_{+}$be the initial positive root system defined in Sect. 1.2. Then other positive root systems are given by $s\left(\Sigma_{+}\right)\left(s \in S_{3}\right)$. Among them, those compatible with the given compact root system $\Sigma_{c,+}$ are $s_{I}\left(\Sigma_{+}\right), s_{I I}\left(\Sigma_{+}\right)$, and $s_{I I I}\left(\Sigma_{-}\right)$. Here $s_{I}=i d, s_{I I}=$ $\left(\begin{array}{lll}1 & 2 & 3 \\ 1 & 3 & 2\end{array}\right), s_{I I I}=\left(\begin{array}{lll}1 & 2 & 3 \\ 3 & 1 & 2\end{array}\right)$.

Let $J$ be a variable taking values in $\{I, I I, I I I\}$. We define a subset $\Xi_{J}$ of the set $L_{T}^{+}=\left\{\Lambda=\left(l_{1}, l_{2}\right) \in \mathbf{Z}^{\oplus 2} \mid l_{1} \geq l_{2}\right\}$ of dominant weights by

$$
\Xi_{J}=\left\{\Lambda=\left(l_{1}, l_{2}\right) \mid\langle\Lambda, \beta\rangle>0 \text { for any root in } s_{J}\left(\Sigma_{+}\right)\right\}
$$

Then

$$
\begin{aligned}
& \Xi_{I}=\left\{\Lambda=\left(l_{1}, l_{2}\right) \mid l_{1}>0, l_{2}>0\right\}, \\
& \Xi_{I I}=\left\{\Lambda=\left(l_{1}, l_{2}\right) \mid l_{1}>0, l_{2}<0\right\},
\end{aligned}
$$

and

$$
\Xi_{I I I}=\left\{\Lambda=\left(l_{1}, l_{2}\right) \mid l_{1}<0, l_{2}<0\right\} .
$$

Then the set $\Xi_{I} \cup \Xi_{I I} \cup \Xi_{I I I}$ parametrizes the discrete series representations of $S U(2,1)$. We may call those representation parametrized by $\Xi_{I}$, holomorphic discrete series, and those parametrized by $\Xi_{I I I}$, antiholomorphic discrete series.

Let $\rho_{c}$ be a half of the sum of compact positive roots; it is given by $\rho_{c}=\frac{1}{2} \beta_{12}$ in our case. Given a Harish-Chandra parameter $\Lambda \in \Xi_{J}$, let $\rho_{n}$ be the half of the sum of non-compact roots in $s_{J}\left(\Sigma_{+}\right)$. The Blattner parameter, namely the highest weight of the minimal $K$-type, of the discrete series representation $\pi_{\Lambda}$ is then given by $\lambda=\Lambda-\rho_{c}+\rho_{n}$.

The Gelfand-Kirillov dimension $G K-\operatorname{dim}\left(\pi_{\Lambda}\right)$ of $\pi_{\Lambda}$ is given by

$$
G K-\operatorname{dim}\left(\pi_{\Lambda}\right)= \begin{cases}2 & \text { if } \Lambda \in \Xi_{I} \cup \Xi_{I I I}, \\ 3 & \text { if } \Lambda \in \Xi_{I I},\end{cases}
$$


(cf. Yamashita [Y-III]). The representations $\pi_{\Lambda}$ with $\Lambda \in \Xi_{I I}$ are hence characterized as large discrete series representations of $G=S U(2,1)$ (cf. Vogan [V]). Since $G$ is quasi-split, the results of $[\mathrm{V}]$ and Kostant $[\mathrm{K}]$ imply that every such representation has a Whittaker model in the $C^{\infty}$ context.

Throughout the following we shall investigate the large discrete series representations of $G=S U(2,1)$. For such a representation, the Harish-Chandra parameter $\Lambda$ coincides with the Blattner parameter $\lambda$. Note that if $\pi_{\lambda}$ is the large discrete series representation with Blattner parameter $\lambda$, then the contragredient of $\pi_{\lambda}$ is also a large discrete series representation, with Blattner parameter $\lambda^{*}$ (cf. Sect. 2.1):

$$
\pi_{\lambda}^{*}=\pi_{\lambda^{*}}
$$

Here is an observation that $K$-types of the (large) discrete series representations of $S U(2,1)$ are multiplicity free, which plays an important role for our later computation of Whittaker functions in $\S 5$. More precisely we have the following.

Proposition (3.1). Let $\left(\pi_{\lambda}, H_{\lambda}\right)$ be the large discrete series representation with Blattner parameter $\lambda$. Then

$$
H_{\lambda}\left|K \cong \oplus_{m, n \geq 0} V_{\lambda+m \beta_{13}+n \beta_{32}}, H_{\lambda}^{*}\right| K \cong \oplus_{m, n \geq 0} V_{\lambda+m \beta_{13}+n \beta_{32}}^{*}
$$

as K-modules.

Proof. This is an immediate consequence from the Blattner formula (cf. [H-S], Theorem (1.3)).

\subsection{Whittaker functionals}

We recall some fundamental results on Whittaker model in this section. Put $N=\exp (\mathfrak{n})$. Let $\eta: N \rightarrow \mathrm{C}^{*}$ be a non-trivial unitary character of $N$. We denote by $C_{\eta}^{\infty}(N \backslash G)$ the space of $C^{\infty}$-functions $\varphi(g)$ on $G$ satisfying.

$$
\varphi(n g)=\eta(n) \varphi(g)
$$

for any $n \in N, g \in G$. By the usual semi-norm system, we equip a structure of Fréchet space on $C_{\eta}^{\infty}(N \backslash G)$. The group $G$ acts continuously on $C_{\eta}^{\infty}(N \backslash G)$ by the right regular action.

Let $(\pi, H)$ be an irreducible unitary representation of $G$, and $H_{K}$ be the $K$-finite vector in $H$. Then we denote by $\operatorname{Hom}_{(\mathrm{g}, K)}\left(\pi, C_{\eta}^{\infty}(N \backslash G)\right)$ the intertwining space from $H_{K}$ to $C_{\eta}^{\infty}(N \backslash G)$ as compatible $(\mathfrak{g}, K)$-modules. This is the space of algebraic Whittaker vectors.

In our case $G=S U(2,1)$. Then $G_{\max }$ given by 


$$
G_{\max }=\left\{g \in G_{\mathrm{c}} \mid \operatorname{Ad}(g) \mathrm{g}=\mathrm{g}\right\},
$$

is equal to $G$ itself. Hence, for $\Lambda \in \Xi_{I} \cup \Xi_{I I} \cup \Xi_{I I}$, the transform of $\pi_{\Lambda}$ with respect to an element $g_{0}$ of $G_{\max }$ is isomorphic to $\pi_{\Lambda}$. Therefore we have

$$
\operatorname{dim}_{\mathbf{C}} \operatorname{Hom}_{(g, K)}\left(\pi_{\Lambda}, C_{\eta}^{\infty}(N \backslash G)\right)=2 \text {, or }=0,
$$

since the order of the (little) Weyl group $W(\mathrm{~g}, \mathfrak{a})$ is 2 (cf. Kostant $[\mathrm{K}]$ ).

Let $\mathscr{A}_{\eta}(N \backslash G)$ be the subspace of $C_{\eta}^{\infty}(N \backslash G)$, consisting of right $K$-finite slowly increasing functions. Then Wallach's variant $[\mathrm{W}, 88]$ of multiplicity one theorem (cf. Shalika $[\mathrm{Sh}]$ ) implies that

$$
\operatorname{dim}_{\mathrm{C}} \operatorname{Hom}_{(\mathrm{g}, K)}\left(\pi_{\Lambda}, \mathscr{A}_{\eta}(N \backslash G)\right)= \begin{cases}1, & \text { if } \Lambda \in \Xi_{I I} ; \\ 0, & \text { if } \Lambda \in \Xi_{I} \cup \Xi_{I I I} .\end{cases}
$$

If $\Lambda=\lambda \in \Xi_{I I}$, we denote by $W h_{\eta}\left(\pi_{\lambda}\right)$ the image of a non-zero element of the above intertwining space.

\subsection{Differential operator $\mathscr{D}_{\eta, \mu}$}

Let $\left(\pi_{\lambda}, H_{\lambda}\right)$ be a large discrete series representation with Blattner parameter $\lambda$. Following Yamashita [Y-I], [Y-II], we identify the intertwining space $\operatorname{Hom}_{(g, K)}\left(\pi_{\lambda}^{*}, C_{\eta}^{\infty}(N \backslash G)\right)$ with a solution space of a partial differential operator.

Let $\left(\pi_{\mu}, V_{\mu}\right)$ be the irreducible $K$-module with highest weight $\mu$. Assume that $\left(\tau_{\mu}^{*}, V_{\mu}^{*}\right)$ occurs in $\pi_{\lambda}^{*}$, namely $\mu \in \lambda+\mathbf{Z}_{\geq 0} \beta_{13}+\mathbf{Z}_{\geq 0} \beta_{32}$ (cf. (3.1)). Fix a $K$ equivariant linear injection $V_{\mu}^{*} \subset H_{\lambda}^{*}$.

Consider the restriction map

$$
W \in \operatorname{Hom}_{(g, K)}\left(\pi_{\lambda}^{*}, C_{\eta}^{\infty}(N \backslash G)\right) \mapsto F_{W} \in \operatorname{Hom}_{K}\left(V_{\mu}^{*}, C_{\eta}^{\infty}(N \backslash G)\right) .
$$

Then $\operatorname{Hom}_{K}\left(V_{\mu}^{*}, C_{\eta}^{\infty}(N \backslash G)\right)=\left\{C_{\eta}^{\infty}(N \backslash G) \otimes V_{\mu}^{*}\right\}^{K}$ is identified with

$$
\begin{aligned}
& C_{\eta, \tau_{\mu}}^{\infty}(N \backslash G / K) \\
& =\left\{\varphi: G \rightarrow V_{\lambda}, C^{\infty} \text {-function } \mid \varphi(n g k)=\eta(n) \tau_{\mu}(k)^{-1} \varphi(g),\right. \\
& \forall(n, g, k) \in N \times G \times K\} .
\end{aligned}
$$

Let $C_{\tau_{\mu}}^{\infty}(G / K)$ denote the space of $V_{\mu}$-valued $C^{\infty}$-functions $\varphi$ on $G$ satisfying

$$
\varphi(g k)=\tau_{\mu}(k)^{-1} \varphi(g) \text { for } g \in G, k \in K .
$$

We define a $\left(V_{\mu} \otimes \mathcal{H}_{\mathrm{c}}\right)$-valued $C^{\infty}$-function $\nabla_{\mu} \varphi$ on $G$ by 


$$
\nabla_{\mu} \varphi(g)=\sum_{\imath=1}^{4} R_{X_{i}} \phi(g) \otimes X_{\imath}
$$

Here, $R_{Y} \phi(g)=\left.\frac{d}{d t} \varphi(g \cdot \exp (t Y))\right|_{t=0}$, and $\left\{X_{i} \mid 1 \leq i \leq 4\right\}$ is an orthonormal basis of $\mathfrak{h}$ with respect to the Killing form of $\mathfrak{g}$. Obviously the map $\nabla_{\mu}$ : $C_{\tau_{\mu}}^{\infty}(G / K) \rightarrow C_{\tau_{\mu} \otimes \text { Ad }}^{\infty}(G / K)$ commutes with $G$-and $g_{\mathrm{c}}$-action from the left. Therefore we have a naturally induced differential operator $\nabla_{\eta, \mu}: C_{\eta, \tau_{\mu}}^{\infty}(N \backslash G / K) \rightarrow$ $C_{\eta, \tau_{\mu} \otimes \mathrm{Ad}}^{\infty}(N \backslash G / K)$. The tensor product $\tau_{\mu} \otimes \mathrm{Ad}=\tau_{\mu} \otimes \operatorname{Ad}_{\mathfrak{p}_{\mathrm{c}}}$ decomposes as

$$
\left(\tau_{\mu} \otimes \mathrm{Ad}_{\mathfrak{p}_{\mathrm{c}}}, V_{\mu} \otimes \mathfrak{p}_{\mathrm{C}}\right) \cong\left(\tau_{\mu}^{+}, V_{\mu}^{+}\right) \oplus\left(\tau_{\mu}^{-}, V_{\mu}^{-}\right),
$$

where

$$
\tau_{\mu}^{+}=\underset{\beta \in s_{I}\left(\Sigma_{-}\right) \cap \Sigma_{n}}{\oplus} \delta(\beta) \cdot \tau_{\mu+\beta}
$$

and

$$
\tau_{\mu}^{-}=\underset{\beta \in s_{u}\left(\Sigma_{-}\right) \cap \Sigma_{n}}{\oplus} \delta(-\beta) \cdot \tau_{\mu-\beta}
$$

with $\delta(\beta)=1$ or $=0$ and $\delta(-\beta)=1$ or $=0$, according as $\mu+\beta$ and $\mu-\beta$ are dominant or not, respectively (cf. Sect. 2.2).

Let $P_{2}(\mu): V_{\mu} \otimes \mathcal{H}_{\mathrm{c}} \rightarrow V_{\mu}^{-}$be the projector to the facter $V_{\mu}^{-}$. Then we set

$$
\mathscr{D}_{\eta, \mu}(\varphi(g))=P_{2}(\mu)\left(\nabla_{\eta, \mu} \varphi(g)\right) \quad\left(\varphi \in C_{\eta, \tau_{\mu}}^{\infty}(N \backslash G / K), g \in G\right) .
$$

Theorem (3.2) ([Y-II, Theorem 2.4]).

Let $\left(\pi_{\lambda}, H_{\lambda}\right)$ be the large discrete series representation of $G$ with Blattner parameter $\lambda$. If $\lambda$ is far from the walls, then the assignment

$$
W \in \operatorname{Hom}_{(\mathrm{g}, K)}\left(\pi_{\lambda}^{*}, C_{\eta}^{\infty}(N \backslash G)\right) \mapsto F_{W} \in \operatorname{Ker}\left(\mathscr{D}_{\eta, \lambda}\right)
$$

is a linear isomorphism.

Remark. As we see later, the assumption "far from the walls" is not necessary for $G=S U(2,1)$, since $\operatorname{dim}_{\mathbf{C}} \operatorname{Ker}\left(\mathscr{D}_{\eta, \lambda}\right)=2$, and $W \mapsto F_{W}$ is injective.

In subsequent sections, we explicitly compute $\mathscr{D}_{\eta, \lambda}$ and $P_{1}(\mu)\left(\nabla_{\eta, \mu} \varphi(*)\right)$ to describe the image of elements in $\operatorname{Hom}_{(\mathrm{g}, K)}\left(\pi_{\lambda}^{*}, C_{\eta}^{\infty}(N \backslash G)\right)$.

\section{$\S 4$. Explicit Formula for the Minimal $K$-type Whittaker Functions}

4.1. Radial part of $\nabla_{\eta, \mu}^{ \pm}$and their projections 
Let $A=\exp (\mathfrak{a})$. In view of the Iwasawa decomposition $G=N A K$, the space $C_{\eta, \tau_{\mu}}^{\infty}(N \backslash G / K)$ is identified with the space $C^{\infty}\left(A, V_{\mu}\right)$ of $C^{\infty}$-functions on $A$ with values in $V_{\mu}$.

We denote by $r_{\eta, \tau_{\mu}}$ and $r_{\eta, \tau_{\mu}^{-}}$the identifications

$$
r_{\eta, \tau}: C_{\eta, \tau_{\mu}}^{\infty}(N \backslash G / K) \stackrel{\sim}{\rightarrow} C^{\infty}\left(A, V_{\mu}\right)
$$

and

$$
r_{\eta, \tau_{\mu}^{-}}: C_{\eta, \tau_{\mu}^{-}}^{\infty}(N \backslash G / K) \stackrel{\sim}{\rightarrow} C^{\infty}\left(A, V_{\mu}^{-}\right) .
$$

Then we define the radial part $R\left(\mathscr{D}_{\eta, \mu}\right)$ of $\mathscr{D}_{\eta, \mu}$ by

$$
R\left(\mathscr{D}_{\eta, \mu}\right)=r_{\eta, \tau_{\mu}^{-}} \circ \mathscr{D}_{\eta, \mu} \circ r_{\eta, \tau_{\mu}}^{-1}: C^{\infty}\left(A, V_{\mu}\right) \rightarrow C^{\infty}\left(A, V_{\mu}^{-}\right)
$$

We want to have an explicit description of $R\left(\mathscr{D}_{\eta, \mu}\right)$.

There is a positive constant $c$ depending on the normalization of the Killing form such that the set

$$
\left\{c\left(X_{\beta_{13}}+X_{\beta_{31}}\right), c \sqrt{-1}\left(X_{\beta_{13}}-X_{\beta_{31}}\right), c\left(X_{\beta_{23}}+X_{\beta_{32}}\right), c \sqrt{-1}\left(X_{\beta_{23}}-X_{\beta_{32}}\right)\right\}
$$

forms an orthonormal basis of $\mathfrak{p}$ with respect to the Killing form. Then

$$
\nabla_{\eta, \mu} F=2 c^{2} \cdot\left(\nabla_{\eta, \mu}^{+}+\nabla_{\eta, \mu}^{-}\right) F
$$

with

$$
\nabla_{\eta, \mu}^{+} F=R_{X_{\beta_{31}}} F \otimes X_{\beta_{13}}+R_{X_{\beta_{32}}} F \otimes X_{\beta_{23}}
$$

and

$$
\nabla_{\eta, \mu}^{-} F=R_{X_{\beta_{13}}} F \otimes X_{\beta_{31}}+R_{X_{\beta_{23}}} F \otimes X_{\beta_{32}}
$$

For $F$ in $C_{\eta, \tau_{\mu}}^{\infty}(N \backslash G / K)$, we denote by $\phi$ its restriction to $A$. We denote by $\partial_{1} \phi$ the restriction of the operator $H_{1}$

$$
\left(H_{1} F\right) \mid A=\partial_{1} \phi \text {. }
$$

We identify $\mathfrak{a}=\mathbf{R} H_{1}$ with $\mathbf{R}$ mapping $H_{1}$ to 1 . Then $A=\exp (\mathfrak{a})$ is identified with $\mathbf{R}_{>0}=\exp (\mathbf{R})=\{r \in \mathbf{R}: r>0\}$. The image of $a \in A$ in $\mathbf{R}_{>0}$ via this identification is denoted by $r(a)$. 


\section{Proposition (4.1).}

Let $\phi$ be an element of $C^{\infty}\left(A, V_{\mu}\right)$. Then the radial part $R\left(\nabla_{\eta, \mu}^{+}\right)$of $\nabla_{\eta, \mu}^{+}$is given by

$$
\begin{aligned}
R\left(\nabla_{\eta, \mu}^{+}\right) \phi= & \frac{1}{2}\left\{\partial_{1}-\sqrt{-1} r(a)^{2} \eta\left(E_{1}\right)-4\right\}\left(\phi \otimes X_{\beta_{13}}\right) \\
& +\frac{1}{2}\left(\tau_{\mu} \otimes \mathrm{Ad}_{\mathfrak{p}_{+}}\right)\left(H_{13}^{\prime}\right)\left(\phi \otimes X_{\beta_{13}}\right) \\
& +\frac{(-1)}{2} r(a)\left\{\eta\left(E_{2,+}\right)-\sqrt{-1} \eta\left(E_{2,-}\right)\right\}\left(\phi \otimes X_{\beta_{23}}\right) \\
& +\left(\tau_{\mu} \otimes \mathrm{Ad}_{\mathfrak{p}_{+}}\right)\left(X_{\beta_{12}}\right)\left(\phi \otimes X_{\beta_{23}}\right) .
\end{aligned}
$$

Similarly for the radial part $R\left(\nabla_{\eta, \mu}^{-}\right)$of $\nabla_{\eta, \mu}^{-}$we have

$$
\begin{aligned}
R\left(\nabla_{\eta, \mu}^{-}\right) \phi= & \frac{1}{2}\left\{\partial_{1}-\sqrt{-1} r(a)^{2} \eta\left(E_{1}\right)-4\right\}\left(\phi \otimes X_{\beta_{31}}\right) \\
& -\frac{1}{2}\left(\tau_{\mu} \otimes \mathrm{Ad}_{\mathfrak{p}_{-}}\right)\left(H_{13}^{\prime}\right)\left(\phi \otimes X_{\beta_{31}}\right) \\
& +\frac{(-1)}{2} r(a)\left\{\eta\left(E_{2,+}\right)+\sqrt{-1} \eta\left(E_{2,-}\right)\right\}\left(\phi \otimes X_{\beta_{32}}\right) \\
& +\left(\tau_{\mu} \otimes \mathrm{Ad}_{\mathfrak{p}_{-}}\right)\left(X_{\beta_{21}}\right)\left(\phi \otimes X_{\beta_{32}}\right) .
\end{aligned}
$$

Proof of Proposition (4.1).

The proof is easy. We indicate only some key points of computation.

When $X \in \mathfrak{f},\left(R_{X} F\right)(a)=-\tau_{\mu}(X) \phi(a)$. Hence for $X \in \mathfrak{l}$ and $Y \in \mathfrak{l}_{\mathrm{c}}$,

$$
\left(R_{X} F\right)(a) \otimes Y=-\left(\tau_{\mu} \otimes \operatorname{Ad}_{p}\right)(X)(\phi(a) \otimes Y)+\phi(a) \otimes[X, Y]
$$

By definition, $R_{H_{1}} F(a)=\partial_{1} \phi(a)$, and simple computations show

$$
R_{E_{1}} F(a)=r(a)^{2} \eta\left(E_{1}\right) \phi(a)
$$

and

$$
R_{H_{13}^{\prime}} F(a) \otimes X_{\beta_{13}}=-\left(\tau_{\mu} \otimes \operatorname{Ad}_{\mathfrak{p}}\right)\left(H_{13}^{\prime}\right)\left(\phi(a) \otimes X_{\beta_{13}}\right)+2\left(\phi(a) \otimes X_{\beta_{13}}\right) .
$$

This shows the part (i) of Proposition. The part (ii) is discussed similarly.

\subsection{The minimal K-type Whittaker functions}

In this subsection, we give an explicit formula of the elements $\phi$ of $C_{\eta, \tau_{\lambda}}^{\infty}(A)$ which comes from $W \in \operatorname{Hom}_{(\mathrm{g}, K)}\left(\pi_{\lambda}^{*}, \mathscr{A}_{\eta}(N \backslash G)\right)$. By (3.2), it satisfies the differential equations $P_{2}(\lambda)\left(R\left(\nabla_{\eta, \lambda}^{ \pm}\right) \phi\right)=0$. Put $d=d_{\lambda}=\lambda_{1}-\lambda_{2}$ and write 


$$
\phi(a)=\sum_{k=0}^{d} c_{k}^{\lambda}(a) v_{k}^{\lambda}
$$

with coefficients $c_{k}^{\lambda}(a)=c_{k}(a)(0 \leq k \leq d)$, where $v_{k}^{\lambda}$,s are the standard of $V_{\lambda}$ (cf. Sect. 2.1). Then we have the following

\section{Proposition (4.2).}

(i) The equation $P_{2}(\lambda)\left(R\left(\nabla_{\eta, \lambda}^{+}\right) \phi\right)=0$ is equivalent to a system of differencedifferential equations:

$\left(C_{2}^{+}\right)_{k}: \quad\left(\partial_{1}-k+\lambda_{2}-2\right) c_{k}(a)+r(a) \eta_{-} \cdot c_{k+1}(a)=0 \quad(0 \leq k \leq d-1)$.

(ii) The equation $P_{2}(\lambda)\left(R\left(\nabla_{\eta, \lambda}^{-}\right) \phi\right)=0$ is equivalent to a system of differencedifferential equations:

$\left(C_{2}^{-}\right)_{k}: \quad\left(\partial_{1}+k-2 d-\lambda_{2}-1\right) c_{k+1}(a)-r(a) \eta_{\perp} \cdot c_{k}(a)=0 \quad(0 \leq k \leq d-1)$.

Here we put

$$
\eta_{ \pm}=\eta\left(E_{2,+}^{\prime}\right) \pm \sqrt{-1} \eta\left(E_{2,-}\right)
$$

Proof. Note first $\left.\eta\right|_{[\pi, n]}=0$, because $\eta$ is a charater. Hence $\eta\left(E_{1}\right)=0$.

We put $X_{\beta_{23}}=w_{0}, X_{\beta_{13}}=w_{1}$. Set $\nu=\lambda-\beta_{32}$. Then,

$$
\begin{aligned}
P_{2}(\lambda)\left(R\left(\nabla_{\eta, \lambda}^{+}\right) \phi\right)= & \frac{1}{2}\left(\partial_{1}-4\right) \sum_{k=0}^{d} c_{k}(a) P_{2}(\lambda)\left(v_{k}^{\lambda} \otimes w_{1}\right) \\
& +\frac{1}{2} \sum_{k=0}^{d} c_{k}(a) \tau_{\nu}\left(H_{13}^{\prime}\right) P_{2}(\lambda)\left(v_{k}^{\lambda} \otimes w_{1}\right) \\
& -\frac{1}{2} r(a) \eta_{-} \sum_{k=0}^{d} c_{k}(a) P_{2}(\lambda)\left(v_{k}^{\lambda} \otimes w_{0}\right) \\
& +\sum_{k=0}^{d} c_{k}(a) \tau_{\nu}\left(X_{\beta_{12}}\right) P_{2}(\lambda)\left(v_{k}^{\lambda} \otimes w_{0}\right) \\
= & -\frac{1}{2} \sum_{k=0}^{d-1}\left(\partial_{1}-4\right) c_{k}(a) v_{k}^{\nu}-\frac{1}{2} \sum_{k=0}^{d-1} c_{k}(a) \tau_{\nu}\left(\frac{H_{12}^{\prime}+Z}{2}\right) v_{k}^{\nu} \\
& -\frac{1}{2} r(a) \eta_{-} \sum_{k=1}^{d} c_{k}(a) v_{k-1}^{\nu}+\sum_{k=1}^{d} c_{k}(a) \tau_{\nu}\left(X_{\beta_{12}}\right) v_{k-1}^{\nu} .
\end{aligned}
$$

Input

$$
\left\{\begin{array}{l}
\tau_{\nu}\left(H_{12}^{\prime}\right) v_{k}^{\nu}=(2 k+1-d) v_{k}^{\nu} \\
\tau_{\nu}(Z) v_{k}^{\nu}=\left(\lambda_{1}+\lambda_{2}+3\right) v_{k}^{\nu}=\left(d+2 \lambda_{2}+3\right) v_{k}^{\nu} \\
\tau_{\nu}\left(X_{\beta_{12}}\right) v_{k-1}^{\nu}=k v_{k}^{\nu}
\end{array}\right.
$$


in the above formula. Then

$$
\begin{aligned}
P_{2}(\lambda)\left(R\left(\nabla_{\eta, \lambda}^{+}\right) \phi\right)= & -\frac{1}{2} \sum_{k=0}^{d-1}\left\{\partial_{1}-4+\left(k+\lambda_{2}+2\right)-2 k\right\} c_{k}(a) v_{k}^{\nu} \\
& -\frac{1}{2} r(a) \eta_{-} \sum_{k=1}^{d} c_{k}(a) v_{k-1}^{\nu},
\end{aligned}
$$

which shows the part (i) of Proposition. The part (ii) is shown in a similar way.

Now via the identification

$$
a=\exp \left(t H_{1}\right) \in A=\exp (\mathfrak{a}) \mapsto r=r(a)=e^{t} \in \mathbf{R}_{>0},
$$

we regard $\phi$ and $e_{k}$ as functions on $\mathbf{R}_{>0}$. Then $\partial_{1}=\frac{d}{d t}=r \frac{d}{d r}$ is the Euler operator in $r$.

Assumption (4.3). We assume that $\eta_{+} \eta_{-} \neq 0$, namely

$$
\eta\left(E_{2,+}\right) \neq 0 \text { or } \eta\left(E_{2,-}\right) \neq 0
$$

Then $\left(C_{+}^{2}\right)_{k}$ and $\left(C_{-}^{2}\right)_{k}$ are equivalent to

$$
\left(C_{+}^{2}\right)_{k}: \quad c_{k+1}(r)=-\eta_{-}^{-1}\left(\frac{d}{d r}+\frac{-k+\lambda_{2}-2}{r}\right) c_{k}(r) \quad(0 \leq k \leq d-1)
$$

and

$$
\left(C_{-}^{2}\right)_{k}: \quad c_{k}(r)=\eta_{+}^{-1}\left(\frac{d}{d r}+\frac{k-2 d-\lambda_{2}-1}{r}\right) c_{k+1}(r) \quad(0 \leq k \leq d-1) .
$$

If we determine $c_{d}(r)$, then by the recurrence formula $\left(C_{-}^{2}\right)_{k}$, we can determine $c_{d-1}(r), \cdots, c_{0}(r)$.

Lemma (4.4). Represent $c_{d-1}, c_{d-2}, \cdots, c_{0}$ downward recurrently by $\left(C_{-}^{2}\right)_{k}$. Then if $\left(C_{+}^{2}\right)_{d-1}$ is satisfied by $c_{d}$ and $c_{d-1}$, then all the $\left(C_{+}^{2}\right)_{k}(0 \leq k \leq d-1)$ are satisfied by $c_{k}(0 \leq k \leq d)$.

Proof. The proof is easy. We omit it.

We want to determine $\left\{c_{k}(r)\right\}_{k=0}^{d}$.

Each $c_{k}(r)$ satisfies a differential equation:

$(D)_{k}:\left[\frac{d^{2}}{d r^{2}}+\frac{(-2 d-3)}{r} \frac{d}{d r}+\left\{\frac{\left(k-2 d-\lambda_{2}-2\right)\left(-k+\lambda_{2}-2\right)}{r^{2}}+\eta_{+} \eta_{-}\right\}\right] c_{k}(r)=0$. 
Noting $-4 \eta_{+} \eta_{-}>0$ for $\eta_{+}$and $\eta_{-}$are purely imaginary numbers, we change the variable $r$ by $x=\sqrt{-4 \eta_{+} \eta_{-}} r$, and set

$$
c_{k}(r)=\left\{\frac{x}{\sqrt{-4 \eta_{+} \eta_{-}}}\right\}^{d+\frac{3}{2}} v_{k}(x) \quad(0 \leq k \leq d) .
$$

Then $v_{k}$ satisfies the Whittaker differential equation.

$(E)_{k}: \quad\left\{\frac{d^{2}}{d x^{2}}+\left(-\frac{1}{4}+\frac{\kappa}{x}+\frac{\frac{1}{4}-m^{2}}{x^{2}}\right)\right\} v_{k}(x)=0$

with $\kappa=0$ and $m^{2}=\left(k-d-\lambda_{2}\right)^{2}=\left(k-\lambda_{1}\right)^{2}$.

Because the image of $C^{-\infty}$-Whittaker functionals should be of moderate growth for $r \rightarrow+\infty$ (cf. Wallach [W]), $v_{k}(x)$ is a constant multiple of the unique solution of $(E)$ :

$$
W_{0, k-\lambda_{1}}(x)=\frac{e^{-\frac{1}{2} x}}{\Gamma\left(\frac{1}{2}+\left|k-\lambda_{1}\right|\right)} \int_{0}^{\infty} t^{-\frac{1}{2}+\left|k-\lambda_{1}\right|}\left(1+\frac{t}{x}\right)^{-\frac{1}{2}+\left|k-\lambda_{1}\right|} e^{-t} d t
$$

with the required growth condition (cf. Magnus-Oberhettinger-Soni [MOS], (Chap. 7). Note that the Whittaker function $W_{\kappa, m}(x)$ reduces to the modified $K$-Bessel function if $\kappa=0$ :

$$
W_{0, m}(x)=\pi^{-1 / 2} x^{1 / 2} K_{m}\left(\frac{x}{2}\right)
$$

Recall that we have the inequalities

$$
\lambda_{1}>0, \quad \lambda_{2}<0, \quad \text { and } d=\lambda_{1}-\lambda_{2}>0
$$

for the parameters. By the above discussion, we may put for $0 \leq k \leq d$

$$
c_{k}(r)=\gamma_{k} r^{d+\frac{3}{2}} W_{0, k-\lambda_{1}}(2 \sqrt{b} r) .
$$

Here $\gamma_{k}$ is a some constant and $b=-\eta_{+} \eta_{-}>0$.

By the recurrence formula

$$
c_{k}(r)=\eta_{+}^{-1}\left(\frac{d}{d r}+\frac{k-2 d-\lambda_{2}-1}{r}\right) c_{k+1}(r) \quad(0 \leq k \leq d-1),
$$

we have 


$$
\begin{aligned}
\eta_{+} \gamma_{k+1}^{-1} c_{k}(r)=(d & \left.+\frac{3}{2}\right) r^{d+\frac{1}{2}} W_{0, k+1-\lambda_{1}}(2 \sqrt{b} r)+2 \sqrt{b} r^{d+\frac{3}{2}} W_{0, k+1-\lambda_{1}}^{\prime}(2 \sqrt{b} r) \\
& +\left(k-2 d-\lambda_{2}-1\right) r^{d+\frac{1}{2}} W_{0, k+1-\lambda_{1}}(2 \sqrt{b} r) \\
& =2 \sqrt{b} r^{d+\frac{3}{2}} \mathscr{L}_{k+1-\lambda_{1}}\left[W_{0, k+1-\lambda_{1}}\right](2 \sqrt{b} r)
\end{aligned}
$$

where we set

$$
\mathscr{L}_{m}[f](z)=\frac{d f}{d z}+\frac{m-\frac{1}{2}}{z} f \text { for } m \in \mathbf{Z} .
$$

Note that the $K$-Bessel functions satisfy

$$
\left(\frac{d}{d z}+\frac{m}{z}\right) K_{m}(z)=-K_{m-1}(z)
$$

(cf. [MOS], Chap.3). We rewrite this relation as

$$
\mathscr{L}_{m}\left[W_{0, m}\right]=-\frac{1}{2} W_{0, m-1}
$$

to get

Theorem (4.5). Normalize the constants $\gamma_{k}$ by $\gamma_{d}=1$. Then for $0 \leq k \leq d$,

$$
c_{k}^{\lambda}(r)=c_{k}(r)=\gamma_{k} r^{d+\frac{3}{2}} W_{0, k-\lambda_{1}}(2 \sqrt{b} r)
$$

with

$$
\begin{gathered}
b=-\eta_{+} \eta_{-}>0 \\
\gamma_{k}=\left(-\frac{\sqrt{b}}{\eta_{+}}\right)^{d-k}=\left(\frac{\eta_{-}}{\sqrt{b}}\right)^{d-k} .
\end{gathered}
$$

\section{§ 5. An Explicit Formula for $K$-finite Whittaker Functions}

In this section, we firstly give some recurrence relations for weight vectors in $K$-type subspaces of $W h_{\eta}\left(\pi_{\lambda}^{*}\right)$, using the fact that $W h_{\eta}\left(\pi_{\lambda}^{*}\right)$ is $K$-multiplicity free. We then translate them into recurrence relations for the Mellin transforms, and give an explicit solution of this translated relations.

\subsection{Recurrence relations for $K$-finite vectors in $W h_{\eta}\left(\pi_{\lambda}^{*}\right)$}

The elements $c_{k}^{\lambda}(*)\left(0 \leq k \leq d_{\lambda}\right)$ of the Whittaker model $W h_{\eta}\left(\pi_{\lambda}^{*}\right)$, to which we have given an explicit formula in the previous section, are the weight vectors 
in the minimal $K$-type subspace. We now investigate the weight vectors in all $K$ type subspaces. Note that the algebraic sum of such vectors coincides with the space $W h_{\eta}\left(\pi_{\lambda}^{*}\right)_{K}$ of $K$-finite vectors.

Let $\mu$ denote, as before, a general element of $\lambda+\mathbf{Z}_{\geq 0} \beta_{13}+\mathbf{Z}_{\geq 0} \beta_{32}$ with

$$
\mu=\left(\mu_{1}, \mu_{2}\right), \quad d_{\mu}=\operatorname{dim}_{\mathrm{C}} V_{\mu}-1\left(=\mu_{1}-\mu_{2}\right) .
$$

Since $\pi_{\lambda}^{*}$ is $K$-multiplicity free (cf. Prop. (3.1)), $W h_{\eta}\left(\pi_{\lambda}^{*}\right)$ contains a unique $K$ invariant subspace which is $K$-isomorphic to $V_{\mu}^{*}$. Call $W h_{\eta}\left(\pi_{\lambda}^{*}\right)_{\mu}^{*}$ this subspace.

We fix a basis of $W h_{\eta}\left(\pi_{\lambda}^{*}\right)_{\mu} *$ which agrees with $\left\{c_{k}^{\lambda}(*) \mid 0 \leq k \leq d_{\lambda}\right\}$ if $\mu=\lambda$. Let $\left\{\left(v_{k}^{\mu}\right)^{*} \mid 0 \leq k \leq d_{\mu}\right\}$ be the basis of $V_{\mu}^{*}$, which is dual to the standard basis $\left\{v_{k}^{\mu} \mid 0 \leq k \leq d_{\mu}\right\}$ of $V_{\mu}$ (cf. Sect. 2.1): $\left\langle v_{i}^{\mu},\left(v_{j}^{\mu}\right)^{*}\right\rangle=\delta_{i j}$. Choose a $K$-isomorphism $V_{\mu}^{*} \cong W h_{\eta}\left(\pi_{\lambda}^{*}\right)_{\mu}$ and let $\left\{c_{k}^{\mu}(*) \mid 0 \leq k \leq d_{\mu}\right\}$ be the image of $\left\{\left(v_{k}^{\mu}\right)^{*} \mid 0 \leq k \leq d_{\mu}\right\}$ under this isomorphism. Each $c_{k}^{\mu}(*)$ is a weight vector of weight $\mu^{*}-k \beta_{12}=$ $-\mu+\left(d_{\mu}-k\right) \beta_{12}$, and the basis $\left\{c_{k}^{\mu}(*) \mid 0 \leq k \leq d_{\mu}\right\}$ of $W h_{\eta}\left(\pi_{\lambda}^{*}\right)_{\mu}^{*}$ is unique up to multiplication by a common non-zero scalar. We denote by $c_{k}^{\mu}(r)$ the restriction of $c_{k}^{\mu}(*)$ to the subgroup $A$ :

$$
c_{k}^{\mu}(r):=c_{k}^{\mu}(a(r))=c_{k}^{\mu}\left(\left(\begin{array}{ccc}
\left(r+r^{-1}\right) / 2 & \left(r-r^{-1}\right) / 2 \\
\left(r-r^{-1}\right) / 2 & 1 & \left(r+r^{-1}\right) / 2
\end{array}\right)\right) .
$$

In $\S \S 2.2$ we have defined the projectors $P_{j}^{ \pm}(\mu)(j=1,2)$ :

$$
\begin{aligned}
& P_{1}^{+}(\mu): V_{\mu} \otimes \mathcal{P}_{\mathrm{c}} \rightarrow V_{\mu+\beta_{13}}, P_{2}^{+}(\mu): V_{\mu} \otimes \mathcal{H}_{\mathrm{c}} \rightarrow V_{\mu-\beta_{32}}, \\
& P_{1}^{-}(\mu): V_{\mu} \otimes \mathcal{H}_{\mathrm{c}} \rightarrow V_{\mu+\beta_{32}}, P_{2}^{-}(\mu): V_{\mu} \otimes \mathcal{H}_{\mathrm{c}} \rightarrow V_{\mu-\beta_{13}} .
\end{aligned}
$$

Proposition (5.1). Let $\partial_{1}$ be the differential operator $r \frac{d}{d r}$ on $\mathbf{R}_{>0}$ and put $c_{-1}^{\mu}(r)=c_{d_{\mu}+1}^{\mu}(r)=0$. One then has, under convention (2.2),

$$
\begin{aligned}
& c_{k+1}^{\mu+\beta_{13}}(r) \sim(k+1)\left(\partial_{1}+\mu_{2}+2 d_{\mu}-k\right) c_{k}^{\mu}(r)-\left(d_{\mu}-k\right) \eta_{-} r c_{k+1}^{\mu}(r)\left(-1 \leq k \leq d_{\mu}\right), \\
& c_{k}^{\mu+\beta_{32}}(r) \sim\left(d_{\mu}-k+1\right)\left(\partial_{1}-\mu_{2}+k\right) c_{k}^{\mu}(r)+k \eta_{+} r c_{k-1}^{\mu}(r)\left(0 \leq k \leq d_{\mu}+1\right) .
\end{aligned}
$$

Proof. Since $W h_{\eta}\left(\pi_{\lambda}^{*}\right)_{K}$ is stable under $U\left(g_{c}\right)$, the correspondence

$$
v^{*} \mapsto<P_{1}^{+}(\mu)\left(\nabla_{\eta, \mu}^{+}\left(\sum_{k} c_{k}^{\mu}(*) v_{k}^{\mu}\right)\right), v^{*}>
$$

defines a $K$-equivariant map from $V_{\mu+\beta_{13}}^{*}$ into $W h_{\eta}\left(\pi_{\lambda}^{*}\right)_{K}$ hence into $W h_{\eta}\left(\pi_{\lambda}^{*}\right)_{\left(\mu+\beta_{13}\right)^{*}}$. We then have, by Schur's lemma,

$$
P_{1}^{+}(\mu)\left(\nabla_{\eta, \mu}^{+}\left(\sum_{0 \leq k \leq d_{\mu} \perp 1} c_{k}^{\mu}(*) v_{k}^{\mu}\right)\right)=\gamma \sum_{0 \leq k \leq d_{\mu}+1} c_{k}^{\mu+\beta_{13}}(*) v_{k}^{\mu+\beta_{13}}
$$


with a constant $\gamma \in \mathbf{C}$, and similarly

$$
P_{1}^{-}(\mu)\left(\nabla_{\eta, \mu}^{-}\left(\sum_{k} c_{k}^{\mu}(*) v_{k}^{\mu}\right)\right)=\delta \sum_{0 \leq k \leq d_{\mu}+1} c_{k}^{\mu+\beta_{32}}(*) v_{k}^{\mu+\beta_{32}}
$$

with a constant $\delta \in \mathbf{C}$. Here we find, from (4.1) and (2.3), that these relations are rewritten as follows (cf. the proof of (4.2)):

(*) the right-hand side of $(1)=\gamma_{0} \gamma c_{k+1}^{\mu+\beta_{13}}(r) \quad\left(-1 \leq k \leq d_{\mu}\right)$,

(**) the right-hand side of $(2)=\delta_{0} \delta c_{k+1}^{\mu \perp \beta_{13}}(r) \quad\left(0 \leq k \leq d_{\mu}+1\right)$.

Here $\gamma_{0}$ and $\delta_{0}$ are non-zero constant independent of $k$. Our task is, hence, to show that $\gamma \delta \neq 0$.

Suppose $\gamma=0$. Then we use (*) firstly with $k=-1$, secondly with $k=0$, and so on, to get firstly $c_{0}^{\mu}(r)=0$, secondly $c_{1}^{\mu}(r)=0$, and so on. Hence a contradiction. We can show $\delta \neq 0$ by a similar argument.

\subsection{The Mellin transforms of $K$-finite Whittaker functions}

Let $\mu$ be an element of $\lambda+\mathbf{Z}_{\geq 0} \beta_{13}+\mathbf{Z}_{\geq 0} \beta_{32}$ and $k$ an integer with $0 \leq k \leq d_{\mu}$. From the explicit formula (4.5) and the recurrence relations (5.1) we see that $c_{k}^{\mu}(r)$ decreases exponentially when $r \rightarrow+\infty$, and that $c_{k}^{\mu}(r)$ remains bounded when $r \rightarrow+0$. We hence may consider its Mellin transform

$$
M_{k}^{\mu}(s):=\int_{0}^{\infty} c_{k}^{\mu}(r) r^{s-1} d r \quad(\operatorname{Re}(s)>0) .
$$

The Mellin transform of the modified Bessel function $W_{0, m}(x)$ is well-known (cf. [MOS], p.91) :

$$
\int_{0}^{\infty} W_{0, m}(x) x^{s-1} d x=\sqrt{\pi}^{-1} 2^{2 s-1} \Gamma\left(\frac{1}{2}\left(s+m+\frac{1}{2}\right)\right) \Gamma\left(\frac{1}{2}\left(s-m+\frac{1}{2}\right)\right) .
$$

Applying this to (4.5) we get, under convention (2.2),

$$
M_{k}^{\lambda}(s) \sim \gamma_{k}\left(\frac{\sqrt{b}}{2}\right)^{-s} \Gamma\left(\frac{1}{2}\left(s-k+2 d_{\lambda}+\lambda_{2}+2\right)\right) \Gamma\left(\frac{1}{2}\left(s+k-\lambda_{2}+2\right)\right) .
$$

Using integration by parts we translate the recurrence relations (5.2) for $c_{k}^{\mu}(r)$ into recurrence relations for $M_{k}^{\mu}(s)$ :

$$
\begin{gathered}
M_{k+1}^{\mu+\beta_{13}}(s) \sim(k+1)\left(s-\mu_{2}-2 d_{\mu}+k\right) M_{k}^{\mu}(s)+\left(d_{\mu}-k\right) \eta_{-} M_{k+1}^{\mu}(s+1), \\
M_{k}^{\mu+\beta_{32}}(s) \sim\left(d_{\mu}-k+1\right)\left(s+\mu_{2}-k\right) M_{k}^{\mu}(s)-k \eta_{+} M_{k-1}^{\mu}(s+1) .
\end{gathered}
$$

The solution of (5.2), (5.3) and (5.4) is given as follows: 
Theorem (5.5). Let $\mu=\lambda+m \beta_{13}+n \beta_{32}\left(m, n \in \mathbf{Z}_{\geq 0}\right)$. For $x \in \mathbf{Z}$ and $p \in \mathbf{Z}_{\geq 0}$, put

$$
[x ; p]=x(x-1) \cdots(x-p+1)(p>0) \text { and }[x ; 0]=1 .
$$

Then $M_{k+m}^{\mu}(s)$ is expressed in the following two ways:

(1)

$$
\text { (1) } \begin{aligned}
& M_{k+m}^{\mu}(s) \sim \gamma_{k}\left(\frac{\sqrt{b}}{2}\right)^{-s} \sum_{p=0}^{n}\left(\begin{array}{l}
n \\
p
\end{array}\right)\left[d_{\lambda}-k+n ; n-p\right][k+m ; p] \\
\times & \Gamma\left(\frac{1}{2}\left(s-k+\lambda_{2}+2\right)\right) \Gamma\left(\frac{1}{2}\left(s-k+\lambda_{2}+2\right)-n+p\right)^{-1} \\
\times & \Gamma\left(\frac{1}{2}\left(s-k-\lambda_{2}+2\right)+p\right) \Gamma\left(\frac{1}{2}\left(s-k-\lambda_{2}+2\right)-m+p\right)^{-1} \\
\times & \Gamma\left(\frac{1}{2}\left(s-k+2 d_{\lambda}+\lambda_{2}+2\right)+p\right) \Gamma\left(\frac{1}{2}\left(s+k-\lambda_{2}+2\right)\right) . \\
& M_{k+m}^{\mu}(s) \sim \gamma_{k}\left(\frac{\sqrt{b}}{2}\right)^{-s} \sum_{p=0}^{m}\left(\begin{array}{l}
m \\
(2)
\end{array}\right)\left[d_{\lambda}-k+n ; p\right][k+m ; m-p] \\
\times & \Gamma\left(\frac{1}{2}\left(s+k-2 d_{\lambda}-\lambda_{2}+2\right)\right) \Gamma\left(\frac{1}{2}\left(s+k-2 d_{\lambda}-\lambda_{2}+2\right)-m+p\right)^{-1} \\
\times & \Gamma\left(\frac{1}{2}\left(s+k+\lambda_{2}+2\right)+p\right) \Gamma\left(\frac{1}{2}\left(s+k+\lambda_{2}+2\right)-n+p\right)^{-1} \\
\times & \Gamma\left(\frac{1}{2}\left(s-k+2 d_{\lambda}+\lambda_{2}+2\right)\right) \Gamma\left(\frac{1}{2}\left(s+k-\lambda_{2}+2\right)+p\right) .
\end{aligned}
$$

From this we immediately have the following

Corollary (5.6). Let $\mathbf{C}[s]$ be the ring of polynomials in $s$ over $\mathbf{C}$.

(1) $\quad M_{k+m+i}^{\lambda+m \beta_{13}+i \beta_{12}}(s) / M_{k+m}^{\lambda+m \beta_{13}}(s) \in \mathbf{C}[s](i \geq 0)$, and

$$
\begin{aligned}
M_{k+m}^{\lambda+m \beta_{13}}(s) & \sim \gamma_{k}\left(\frac{\sqrt{b}}{2}\right)^{-s} \Gamma\left(\frac{1}{2}\left(s-k-\lambda_{2}+2\right)\right) \Gamma\left(\frac{1}{2}\left(s-k-\lambda_{2}+2\right)-m\right)^{-1} \\
\times & \Gamma\left(\frac{1}{2}\left(s-k+2 d_{\lambda}+\lambda_{2}+2\right)\right) \Gamma\left(\frac{1}{2}\left(s+k-\lambda_{2}+2\right)\right) . \\
(2) & M_{k+i}^{\lambda+n \beta_{32}+\imath \beta_{12}}(s) / M_{k}^{\lambda+n \beta_{32}}(s) \in \mathbf{C}[s](i \geq 0), \text { and } \\
M_{k}^{\lambda+n \beta_{32}}(s) & \sim \gamma_{k}\left(\frac{\sqrt{b}}{2}\right)^{-s} \Gamma\left(\frac{1}{2}\left(s+k+\lambda_{2}+2\right)\right) \Gamma\left(\frac{1}{2}\left(s+k+\lambda_{2}+2\right)-n\right)^{-1} \\
\times & \Gamma\left(\frac{1}{2}\left(s-k+2 d_{\lambda}+\lambda_{2}+2\right)\right) \Gamma\left(\frac{1}{2}\left(s+k-\lambda_{2}+2\right)\right) .
\end{aligned}
$$


(3) $\quad M_{i}^{\lambda+m \beta_{13}+n \beta_{32}+\imath \beta_{12}}(s) / M_{0}^{\lambda+m \beta_{13}+n \beta_{32}}(s) \in \mathbf{C}[s](i \geq 0)$, and

$$
\begin{aligned}
& M_{0}^{\lambda+m \beta_{13}+n \beta_{12}}(s) \sim \gamma_{-m}\left(\frac{\sqrt{b}}{2}\right)^{-s} \Gamma\left(\frac{1}{2}\left(s+m+\lambda_{2}+2\right)\right) \Gamma\left(\frac{1}{2}\left(s+m+\lambda_{2}+2\right)-n\right)^{-1} \\
& \times \Gamma\left(\frac{1}{2}\left(s+m+2 d_{\lambda}+\lambda_{2}+2\right)\right) \Gamma\left(\frac{1}{2}\left(s+m-\lambda_{2}+2\right)\right) . \\
&(4) \quad M_{d_{\lambda}+m+n+i}^{\lambda+m \beta_{13}+n \beta_{32}+2 \beta_{12}}(s) / M_{d_{\lambda}+m+n}^{\lambda+m \beta_{13}+n \beta_{32}}(s) \in \mathbf{C}[s](i \geq 0), \text { and } \\
& M_{d_{\lambda}+m+n}^{\lambda+m \beta_{13}+n \beta_{32}}(s) \sim \gamma_{d_{\lambda}+n}\left(\frac{\sqrt{b}}{2}\right)^{-s} \\
& \times \Gamma\left(\frac{1}{2}\left(s+n-d_{\lambda}-\lambda_{2}+2\right)\right) \Gamma\left(\frac{1}{2}\left(s+n-d_{\lambda}-\lambda_{2}+2\right)-m\right)^{-1} \\
& \times \Gamma\left(\frac{1}{2}\left(s+n+d_{\lambda}+\lambda_{2}+2\right)\right) \Gamma\left(\frac{1}{2}\left(s+n+d_{\lambda}-\lambda_{2}+2\right)\right) .
\end{aligned}
$$

In the following we prove (5.5).

Proof of (5.5) (1) the case $n=0$ and (5.5) (2) in the case $m=0$. In the case $n=0,(5.5)$ (1) reduces to

$$
\begin{aligned}
M_{k+m}^{\mu}(s)=M_{k+m}^{\lambda+m \beta_{13}}(s) & =\gamma_{k}\left(\frac{\sqrt{b}}{2}\right)^{-s} \Gamma\left(\frac{1}{2}\left(s-k-\lambda_{2}+2\right)\right) \Gamma\left(\frac{1}{2}\left(s-k-\lambda_{2}+2\right)-m\right)^{-1} \\
& \times \Gamma\left(\frac{1}{2}\left(s-k+2 d_{\lambda}+\lambda_{2}+2\right)\right) \Gamma\left(\frac{1}{2}\left(s+k-\lambda_{2}+2\right)\right),
\end{aligned}
$$

and in the case $m=0,(5.5)$ (2) reduces to

$$
\begin{aligned}
M_{k}^{\mu}(s)=M_{k}^{\lambda+n \beta_{32}}(s)=\gamma_{k}\left(\frac{\sqrt{b}}{2}\right)^{-s} \Gamma\left(\frac{1}{2}\left(s+k+\lambda_{2}+2\right)\right) \Gamma\left(\frac{1}{2}\left(s+k+\lambda_{2}+2\right)-n\right)^{-1} \\
\times \Gamma\left(\frac{1}{2}\left(s-k+2 d_{\lambda}+\lambda_{2}+2\right)\right) \Gamma\left(\frac{1}{2}\left(s+k-\lambda_{2}+2\right)\right) .
\end{aligned}
$$

(cf. (5.6) (1), (2)). Suppose that the functions $M_{k+m}^{\lambda+m \beta_{13}}(s)$ and $M_{k}^{\lambda+n \beta_{32}}(s)$ are given by these formulas. Then they clearly satisfy (5.2). We also see

$$
\begin{aligned}
& \{(k+m)+1\}\left\{s-\left(\lambda_{2}+m\right)-2\left(d_{\lambda}+m\right)+(k+m)\right\} M_{k+m}^{\lambda+m \beta_{13}}(s) \\
& +\left\{\left(d_{\lambda}+m\right)-(k+m)\right\} \eta_{-} M_{k+m+1}^{\lambda+m \beta_{13}}(s+1) \\
= & (k+m+1)\left(s+k-2 d_{\lambda}-\lambda_{2}-2 m\right) M_{k+m}^{\lambda+m \beta_{13}}(s) \\
& +\eta_{-}\left(\gamma_{k+1} / \gamma_{k}\right)\left(\frac{\sqrt{b}}{2}\right)^{-1}\left(d_{\lambda}-k\right) \frac{1}{2}\left(s+k-\lambda_{2}+2\right) M_{k+m}^{\lambda+m \beta_{13}}(s)
\end{aligned}
$$




$$
\begin{aligned}
& =\left\{(k+m+1)\left(s+k-2 d_{\lambda}-\lambda_{2}-2 m\right)+\left(d_{\lambda}-k\right)\left(s+k-\lambda_{2}+2\right)\right\} M_{k+m}^{\lambda+m \beta_{13}}(s) \\
& =\left(d_{\lambda}+m+1\right)\left(s-k-\lambda_{2}-2 m\right) M_{k+m}^{\lambda+m \beta_{13}}(s)
\end{aligned}
$$

(note that $\eta_{-} \gamma_{k+1}=\gamma_{k} \sqrt{b}$ ). Up to a non-zero constant independent of $k$, the last expression is equal to $M_{k+m+1}^{\lambda+(m+1) \beta_{13}}(s)$. We hence have shown the first recurrence relation (5.3). Similarly, we have

$$
\begin{aligned}
\left\{\left(d_{\lambda}+n\right)-k+\right. & 1\}\left\{s+\left(\lambda_{2}-2 n\right)-k\right\} M_{k}^{\lambda+n \beta_{32}}(s)-k \eta_{+} M_{k-1}^{\lambda+n \beta_{32}}(s+1) \\
= & \left(d_{\lambda}+n-k+1\right)\left(s-k+\lambda_{2}-2 n\right) M_{k}^{\lambda+n \beta_{32}}(s) \\
& -k \eta_{-}\left(\gamma_{k-1} / \gamma_{k}\right)\left(\frac{\sqrt{b}}{2}\right)^{-1} 2\left(s-k+2 d_{\lambda}+\lambda_{2}+2\right) M_{k}^{\lambda+n \beta_{32}}(s) \\
= & \left\{\left(d_{\lambda}+n-k+1\right)\left(s-k+\lambda_{2}-2 n\right)+k\left(s-k+2 d_{\lambda}+\lambda_{2}+2\right)\right\} M_{k}^{\lambda+n \beta_{32}}(s) \\
= & \left(d_{\lambda}+n+1\right)\left(s+k+\lambda_{2}-2 n\right) M_{k}^{\lambda+n \beta_{32}}(s)
\end{aligned}
$$

(note that $-\eta_{+} \gamma_{k-1}=\gamma_{k} \sqrt{b}$ ). Up to a non-zero constant independent of $k$, the last expression is equal to $M_{k}^{\lambda+(n+1) \beta_{32}}(s)$. We hence have (5.4).

Proof of (5.5) (general case). We prove (5.5) (1) by induction in $n$ ((2) is proved by induction in $m$, in the same way). We have done the case $n=0$ in the above. Suppose that (5.5) (1) is true for $n$. By recurrence formula (5.4) we then have,

$$
\begin{aligned}
M_{k+m}^{\lambda+m \beta_{13}+(n+1) \beta_{32}}(s) & \sim\left(d_{\lambda}-k+n+1\right)\left(s-k+\lambda_{2}-2 n\right) M_{k+m}^{\lambda+m \beta_{13}+n \beta_{32}}(s) \\
& -(k+m) \eta_{+} M_{k+m-1}^{\lambda+m \beta_{13}+n \beta_{32}}(s+1) .
\end{aligned}
$$

The assumption of induction, together with the relation $(x+1)[x ; p]=$ $[x+1 ; p+1]$, implies

$$
\begin{aligned}
& \left(d_{\lambda}-k+n+1\right)\left(s-k+\lambda_{2}-2 n\right) M_{k^{+}}^{\lambda+m \beta_{13}+n \beta_{32}}(s) \\
& =\gamma_{k}\left(\frac{\sqrt{b}}{2}\right)^{-s} \sum_{p=0}^{n}\left(\begin{array}{l}
n \\
p
\end{array}\right)\left[d_{\lambda}-k+n+1 ; n+1-p\right][k+m ; p]\left(s-k+\lambda_{2}-2 n\right) \\
& \quad \times \Gamma\left(\frac{1}{2}\left(s-k+\lambda_{2}+2\right)\right) \Gamma\left(\frac{1}{2}\left(s-k+\lambda_{2}+2\right)-n+p\right)^{-1} \\
& \quad \times \Gamma\left(\frac{1}{2}\left(s-k-\lambda_{2}+2\right)+p\right) \Gamma\left(\frac{1}{2}\left(s-k-\lambda_{2}+2\right)-m+p\right)^{-1} \\
& \quad \times \Gamma\left(\frac{1}{2}\left(s-k+2 d_{\lambda}+\lambda_{2}+2\right)+p\right) \Gamma\left(\frac{1}{2}\left(s+k-\lambda_{2}+2\right)\right) .
\end{aligned}
$$

Noting $-\eta_{+} \gamma_{k-1}=\gamma_{k} \sqrt{b}$, we similarly have 


$$
\begin{aligned}
& -(k+m) \eta_{+} M_{k+m-1}^{\lambda+m \beta_{13}+n \beta_{32}}(s+1) \\
& =\gamma_{k}\left(\frac{\sqrt{b}}{2}\right)^{-s} \sum_{p=0}^{n}\left(\begin{array}{l}
n \\
p
\end{array}\right)\left[d_{\lambda}-k+n+1 ; n-p\right][k+m ; p+1]\left(s-k+\lambda_{2}+2\right) \\
& \quad \times \Gamma\left(\frac{1}{2}\left(s-k+\lambda_{2}+2\right)\right) \Gamma\left(\frac{1}{2}\left(s-k+\lambda_{2}+2\right)-n+p+1\right)^{-1} \\
& \quad \times \Gamma\left(\frac{1}{2}\left(s-k-\lambda_{2}+2\right)+p\right) \Gamma\left(\frac{1}{2}\left(s-k-\lambda_{2}+2\right)-m+p+1\right)^{-1} \\
& \quad \times \Gamma\left(\frac{1}{2}\left(s-k+2 d_{\lambda}+\lambda_{2}+2\right)+p+1\right) \Gamma\left(\frac{1}{2}\left(s+k-\lambda_{2}+2\right)\right) .
\end{aligned}
$$

We thus get

$$
M_{k+m}^{\lambda+m \beta_{13}+(n+1) \beta_{32}}(s)
$$

$$
\sim \gamma_{k}\left(\frac{\sqrt{b}}{2}\right)^{-s} \sum_{p=0}^{n+1} A_{p} \Gamma\left(\frac{1}{2}\left(s-k+\lambda_{2}+2\right)\right) \Gamma\left(\frac{1}{2}\left(s+k-\lambda_{2}+2\right)\right)
$$

with

$$
\begin{aligned}
A_{0}= & {\left[d_{\lambda}-k+n+1 ; n+1\right]\left(s-k+\lambda_{2}-2 n\right) \Gamma\left(\frac{1}{2}\left(s-k+\lambda_{2}+2\right)-n\right)^{-1} } \\
& \times \Gamma\left(\frac{1}{2}\left(s-k-\lambda_{2}+2\right)\right) \Gamma\left(\frac{1}{2}\left(s-k-\lambda_{2}+2\right)-m\right)^{-1} \Gamma\left(\frac{1}{2}\left(s-k+2 d_{\lambda}+\lambda_{2}+2\right)\right) \\
= & 2\left[d_{\lambda}-k+n+1 ; n+1\right] \Gamma\left(\frac{1}{2}\left(s-k+\lambda_{2}+2\right)-n-1\right)^{-1} \Gamma\left(\frac{1}{2}\left(s-k-\lambda_{2}+2\right)\right) \\
& \times \Gamma\left(\frac{1}{2}\left(s-k-\lambda_{2}+2\right)-m\right)^{-1} \Gamma\left(\frac{1}{2}\left(s-k+2 d_{\lambda}+\lambda_{2}+2\right)\right), \\
A_{p}= & {\left[d_{\lambda}-k+n+1 ; n+1-p\right][k+m ; p] } \\
& \times\left\{\left(\begin{array}{l}
n \\
p
\end{array}\right)\left(s-k+\lambda_{2}-2 n\right)+\left(\begin{array}{c}
n \\
p-1
\end{array}\right)\left(s-k+\lambda_{2}+2\right)\right\} \\
& \times \Gamma\left(\frac{1}{2}\left(s-k+\lambda_{2}+2\right)-n+p\right)^{-1} \Gamma\left(\frac{1}{2}\left(s-k-\lambda_{2}+2\right)+p\right) \\
& \times \Gamma\left(\frac{1}{2}\left(s-k-\lambda_{2}+2\right)-m+p\right)^{-1} \Gamma\left(\frac{1}{2}\left(s-k+2 d_{\lambda}+\lambda_{2}+2\right)+p\right) \quad(1 \leq p \leq n),
\end{aligned}
$$




$$
\begin{aligned}
A_{n+1}= & {[k+m ; n+1]\left(s-k+\lambda_{2}+2\right) } \\
& \times \Gamma\left(\frac{1}{2}\left(s-k+\lambda_{2}+2\right)+1\right)^{-1} \Gamma\left(\frac{1}{2}\left(s-k-\lambda_{2}+2\right)+n+1\right) \\
& \times \Gamma\left(\frac{1}{2}\left(s-k-\lambda_{2}+2\right)-m+n+1\right)^{-1} \Gamma\left(\frac{1}{2}\left(s-k+2 d_{\lambda}+\lambda_{2}+2\right)+n+1\right) \\
= & 2[k+m ; n+1] \Gamma\left(\frac{1}{2}\left(s-k+\lambda_{2}+2\right)\right)^{-1} \Gamma\left(\frac{1}{2}\left(s-k-\lambda_{2}+2\right)+n+1\right) \\
& \times \Gamma\left(\frac{1}{2}\left(s-k-\lambda_{2}+2\right)-m+n+1\right)^{-1} \Gamma\left(\frac{1}{2}\left(s-k+2 d_{\lambda}+\lambda_{2}+2\right)+n+1\right) .
\end{aligned}
$$

We hence find that the terms for $p=0$ and $p=n+1$ in (*) are twice of the desired terms for $p=0$ and $p=n+1$ for $M_{k^{+} m}^{\lambda+m \beta_{13} \perp(n+1) \beta_{32}}(s)$, respectively. As for the terms for $1 \leq p \leq n$ we use the equality

$$
\left(\begin{array}{l}
n \\
p
\end{array}\right)\left(s-k+\lambda_{2}-2 n\right)+\left(\begin{array}{c}
n \\
p-1
\end{array}\right)\left(s-k+\lambda_{2}+2\right)=\left(\begin{array}{c}
n+1 \\
p
\end{array}\right)\left(s-k+\lambda_{p}-2 n+2 p\right),
$$

and find that the term for $p$ in $(*)$ is twice of the desired term. This completes the proof.

\subsection{The case of $U(2,1)$}

We denote by $\tilde{G}$ the real reductive group $U(2,1)=U\left(I_{2,1}\right)$. It has the semisimple part $G=S U(2,1)$ and the center $Z \tilde{G} \simeq \mathbb{C}^{(1)}$. An irreducible representation of $\tilde{G}$ is determined by its restriction to $G$ and its "central character".

Let $\pi_{\lambda}$ be, as before, a large discrete series representation of $G$ with the Blattner parameter $\lambda=\left(\lambda_{1}, \lambda_{2}\right)\left(\lambda_{1}>0, \lambda_{2}<0\right)$. Its "central character" is given by

$$
\zeta \cdot 1_{3} \rightarrow \zeta^{\lambda_{1}+\lambda_{2}} \quad\left(\zeta \in \mathbf{C}^{(1)}, \zeta^{3}=1\right)
$$

Suppose given an irreducible representation $\tilde{\pi}$ of $\tilde{G}$ with $\tilde{\pi} \mid G=\pi_{\lambda}$. We shall investigate the contragradient $\tilde{\pi}^{*}$ rather than $\tilde{\pi}$ itself. The "central character" of $\tilde{\pi}^{*}$ is given by

$$
\zeta \cdot 1_{3} \rightarrow \zeta^{-\left(\lambda_{1}+\lambda_{2}\right)+3 \nu} \quad\left(\zeta \in \mathbf{C}^{(1)}\right)
$$

with an integer $\nu$, and the datum $(\lambda, \nu)$ determines $\tilde{\pi}^{*}$ completely. We put $\tilde{\pi}^{*}=\tilde{\pi}_{\lambda, \nu}^{*}$. The representation space of $\tilde{\pi}_{\lambda, \nu}^{*}$ remains to be $H_{\lambda}^{*}$, and the smooth vectors of $\tilde{\pi}_{\lambda, \nu}^{*}$ remains to be $\left(H_{\lambda}^{*}\right)^{\infty}$.

The (smooth) Whittaker functional for $\left(\tilde{\pi}_{\lambda, \nu}^{*}, \eta\right)$ is , by definition, a continuous linear functional $L$ on $\left(H_{\lambda}^{*}\right)^{\infty}$ satisfying 


$$
L\left(\tilde{\pi}_{\lambda, \nu}^{*}(n) v^{*}\right)=\eta(n) L\left(v^{*}\right) \quad\left(n \in N, v^{*} \in\left(H_{\lambda}^{*}\right)^{\infty}\right) .
$$

Since $\left.\tilde{\pi}_{\lambda, \nu}^{*}\right|_{N}=\pi_{\lambda ! N}^{*}$, this is nothing but the (smooth) Whittaker functional for $\left(\pi_{\lambda}^{*}, \eta\right)$. On the other hand, the $K$-finite members $\tilde{W}$ (resp. $W$ ) of the smooth Whittaker model $W h_{\eta}\left(\tilde{\pi}_{\lambda, \nu}^{*}\right)$ (resp. $\left.W h_{\eta}\left(\pi_{\lambda}^{*}\right)\right)$ are in one-to-one correspondence with $K$-finite members $v^{*}$ of $\left(H_{\lambda}^{*}\right)^{\infty}$ via

$$
\tilde{W}(g)=L\left(\tilde{\pi}_{\lambda, \nu}^{*}(g) v^{*}\right) \quad\left(\operatorname{resp} . W(g)=L\left(\pi_{\lambda}^{*}(g) v^{*}\right)\right) .
$$

We hence get

Proposition (5.7). The K-finite Whittaker functions $\tilde{W}$ in $W h_{\eta}\left(\tilde{\pi}_{\lambda, \nu}^{*}\right)$ and the $K$-finite Whittaker functions $W$ in $W h_{\eta}\left(\pi_{\lambda}^{*}\right)$ are in one-to-one correspondence via

$$
\tilde{W}(\zeta g)=\zeta^{-\left(\lambda_{1}+\lambda_{2}\right)-3 \nu} W(g) \quad\left(\zeta \in \mathbf{C}^{(1)}, g \in G\right) .
$$

This correspondence gives an K-isomorphism between $W h_{\eta}\left(\tilde{\pi}_{\lambda, \nu}^{*}\right)_{K}$ and $W h_{\eta}\left(\pi_{\lambda}^{*}\right)_{K}$.

\section{§6. The Local Zeta Integrals and the Gamma Factor}

Gelbart and Piatetski-Shapiro [G-Ps] attached certain zeta integrals to a generic representation of the quasi-split unitary group in three variables, defined over a local field. In this section we shall evaluate these integrals in the case where the base field is real and the representation belongs to the large discrete series of $U(2,1)$. We shall see that the "greatest common divisor" of the integrals turns out to be a product of three gamma functions.

\subsection{The Mellin transforms $F_{\Phi}^{\omega}(h ; s)$}

Let $\tilde{H}$ be the real reductive group $U(1,1)=U\left(I_{1,1}\right)$ and $H$ its semisimple part $S U(1,1)=S U\left(I_{1,1}\right)$. There are several subgroups of $\tilde{H}$ to be listed:

$$
\begin{aligned}
& Z(\tilde{H}):=\left\{e^{i \varphi} \cdot 1_{2} \mid \varphi \in \mathbf{R}\right\}, \\
& K^{\prime}:=\left\{\left(\begin{array}{cc}
e^{i \theta} & 0 \\
0 & e^{-i \theta}
\end{array}\right) \mid \theta \in \mathbf{R}\right\}, \\
& A:=\left\{\left(\begin{array}{cc}
\left(r+r^{-1}\right) / 2 & \left(r-r^{-1}\right) / 2 \\
\left(r-r^{-1}\right) / 2 & \left(r+r^{-1}\right) / 2
\end{array}\right) \mid r \in \mathbf{R}\right\}, \\
& Z(N):=\left\{\left(\begin{array}{cc}
1+\frac{i}{2} b & -\frac{i}{2} b \\
\frac{i}{2} b & 1-\frac{i}{2} b
\end{array}\right) \mid b \in \mathbf{R}\right\} .
\end{aligned}
$$


Then $Z(\tilde{H}) K^{\prime}$ is a maximal compact subgroup of $\tilde{H}$, which is also a compact Cartan subgroup of the reductive group $\tilde{H}$.

The group $\tilde{H}$ naturally acts on $W:=\mathbf{C}^{2}$ from the left. Let $\mathscr{S}(W)$ be the Schwartz space on $W$. Fix the left action of $\tilde{H}$ on $\mathscr{S}(W)$ via

$$
(h \Phi)(w):=\Phi\left(h^{-1} \cdot w\right) \quad(h \in \tilde{H}, \Phi \in \mathscr{S}(W), w \in W) .
$$

Fix an isotropic vector $\ell$ in the Hermitian space $\left(W, I_{1,1}\right)$ by

$$
\ell:=\left(\begin{array}{l}
1 \\
1
\end{array}\right)
$$

We also fix a quasi-character $\omega: \mathbf{C}^{\times} \rightarrow \mathbf{C}^{\times}$. It has two parameters $\omega_{0} \in \mathbf{C}$ and $\omega_{1} \in \mathbf{Z}$ :

$$
\omega(t)=|t|_{\mathbf{C}}^{\omega_{0}}\left(\frac{t}{|t|}\right)^{\omega_{1}} \quad\left(t \in \mathbf{C}^{\times}\right)
$$

The Mellin transform $F_{\Phi}^{\omega}(h ; s)$ of $\Phi \in \mathscr{S}(W)$, which was introduced in [G-Ps], is defined by

$$
F_{\Phi}^{\omega}(h ; s):=\int_{\mathbf{C}^{\times}}(h \Phi)(t \ell) \omega(t)|t|_{\mathbf{C}}^{s} d^{\times} t \quad(h \in \tilde{H}, s \in \mathbf{C})
$$

where $d^{\times} t=d t /|t|_{\mathbf{C}}$ is the normalized Haar measure on $\mathbf{C}^{\times}$.

For a 4-tuple $p=\left(m_{1}, n_{1}, m_{2}, n_{2}\right)$ of non-negative integers, define an element $\Phi_{p}$ of $\mathscr{S}(W)$ by

$$
\Phi_{p}(w):=z_{1}^{m_{1}} \bar{z}_{1}^{n_{1}} \exp \left(-\frac{1}{2}\left|z_{1}\right|^{2}\right) z_{2}^{m_{2}} \bar{z}_{2}^{n_{2}} \exp \left(-\frac{1}{2}\left|z_{2}\right|^{2}\right) \quad\left(w=\left(\begin{array}{l}
z_{1} \\
z_{2}
\end{array}\right) \in W\right) .
$$

Then the linear span of the subset $\left\{\Phi_{p}\right\}_{p}$ is dense in $\mathscr{S}(W)$ (cf. [RS], Appendix to V.3). We evaluate the above Mellin transform at $\Phi=\Phi_{p}$.

Using the Iwasawa decomposition $\tilde{H}=Z(\tilde{H}) Z(N) A K^{\prime}$ we write $h \in \tilde{H}$ as

(*) $\quad h=e^{i \varphi}\left(\begin{array}{cc}1+\frac{i}{2} b & -\frac{i}{2} b \\ \frac{i}{2} & 1-\frac{i}{2} b\end{array}\right)\left(\begin{array}{cc}\left(r+r^{-1}\right) / 2 & \left(r-r^{-1}\right) / 2 \\ \left(r-r^{-1}\right) / 2 & \left(r+r^{-1}\right) / 2\end{array}\right)\left(\begin{array}{cc}e^{i \theta} & 0 \\ 0 & e^{i \theta}\end{array}\right)$.

We then have, for $p=\left(m_{1}, n_{1}, m_{2}, n_{2}\right)$, 


$$
\begin{aligned}
\left(h \Phi_{p}\right)(t \ell)= & \exp \left(\left(n_{1}-m_{1}+n_{2}-m_{2}\right) i \varphi+\left(n_{1}-m_{1}-n_{2}+m_{2}\right) i \theta\right) \\
& \times\left(\frac{t}{r}\right)^{m_{1}+m_{2}}\left(\frac{\bar{t}}{r}\right)^{n_{1}+n_{2}} \exp \left(-\left(\frac{|t|}{r}\right)^{2}\right) .
\end{aligned}
$$

Using the polar coordinates $t=\rho e^{i \xi}$ and the change of variable $\frac{\rho}{r}=x$, we find

$$
\begin{aligned}
F_{\Phi_{p}}^{\omega}(h ; s)= & \exp \left(\left(n_{1}-m_{1}+n_{2}-m_{2}\right) i \varphi+\left(n_{1}-m_{1}-n_{2}+m_{2}\right) i \theta\right) r^{2\left(s+\omega_{0}\right)} \\
& \times \int_{0}^{\infty} x^{2\left(s+\omega_{0}\right)+m_{1}+m_{2}+n_{1}+n_{2}} \exp \left(-x^{2}\right) d x / x \\
& \times \int_{0}^{\infty} \exp \left(\left(m_{1}+m_{2}-n_{1}-n_{2}+\omega_{1}\right) i \xi\right) d \xi .
\end{aligned}
$$

We thus have the following

Lemma (6.2). Let $h \in \tilde{H}$ be as in (*) and $p=\left(m_{1}, n_{1}, m_{2}, n_{2}\right)$. Then

(1) $F_{\Phi_{p}}^{\omega}(h ; s)=0$ unless $\omega_{1}=n_{1}+n_{2}-m_{1}-m_{2}$.

(2) In the case $\omega_{1}=n_{1}+n_{2}-m_{1}-m_{2}$ one has

$$
F_{\Phi_{p}}^{\omega}(h ; s)=c_{0} \exp \left(\omega_{1} i \varphi+\left(n_{1}-m_{1}-n_{2}+m_{2}\right) i \theta\right) r^{2\left(s+\omega_{0}\right)} \Gamma\left(s+\omega_{0}-\frac{1}{2} \omega_{1}+n_{1}+n_{2}\right)
$$

where $c_{0}$ is an absolute constant different from 0.

6.2. The local zeta integrals $L^{\omega}(s, \tilde{W}, \Phi)$ and their greatest common divisor The group $\tilde{H}=U(1,1)$ is embedded in $\tilde{G}=U(2,1)$ by

$$
\tilde{H} \ni\left(\begin{array}{cc}
\alpha & \beta \\
\gamma & \delta
\end{array}\right) \mapsto\left(\begin{array}{ccc}
\alpha & 0 & \beta \\
0 & 1 & 0 \\
\gamma & 0 & \delta
\end{array}\right) \in \tilde{G}
$$

The groups $Z(\tilde{H}), K^{\prime}, \tilde{A}$ and $Z(N)$ are thus regarded as subgroups of $\tilde{G}$. We parametrize the elements of the first three groups as follows:

$$
\begin{aligned}
& z(\varphi):=\left(\begin{array}{ccc}
e^{i \varphi} & & \\
& 1 & \\
& & e^{i \varphi}
\end{array}\right), a(r):=\left(\begin{array}{lll}
\left(r+r^{-1}\right) / 2 & & \left(r-r^{-1}\right) / 2 \\
& 1 & \\
& & \\
& & \\
& &
\end{array}\right), \\
& k^{\prime}(\theta):=\left(\begin{array}{ccc}
e^{i \theta} & & \\
& 1 & \\
& & e^{-i \theta}
\end{array}\right)
\end{aligned}
$$


The local zeta integral of Gelbart and Piatetski-Shapiro (in the Archimedean case) is defined by

$$
L^{\omega}(s, \tilde{W}, \Phi)=\int_{Z(N) \backslash \tilde{H}} \tilde{W}(h) F_{\Phi}^{\omega}(h ; s) d h \quad(\Phi \in \mathscr{S}(W)),
$$

with a Whittaker function $\tilde{W}$ on $\tilde{G}$ which belongs to a generic representation (cf. [G-Ps]). We evaluate this integral for our $K$-finite Whittaker functions $\tilde{W}$ which belong to the large discrete series representation $\tilde{\pi}_{\lambda, \nu}^{*}$ (cf. Sect. 5.3).

From now on we assume, without any loss of generality, that $\tilde{W} \in W h_{\eta}\left(\tilde{\pi}_{\lambda, \nu}^{*}\right)$ is a weight vector contained in a $K$-type subspace. If we denote the weight of $\tilde{W}$ by $-\rho$, then $\rho$ belongs to the set of weights of $\pi_{\lambda}$ (cf. Sect. 3 ). Hence we may put

$$
\begin{aligned}
& \text { the weight of } \tilde{W}=-\rho \text {, } \\
& \rho=\lambda+e \beta_{13}+f \beta_{32}=\left(\lambda_{1}+2 e-f, \lambda_{2}+e-2 f\right) \quad\left(e, f \in \mathbf{Z}_{\geq 0}\right) .
\end{aligned}
$$

Lemma (6.4). The above $\tilde{W}$ satisfies

$$
\begin{aligned}
& \tilde{W}\left(z(\varphi) n a(r) k^{\prime}(\theta)\right) \\
& \quad=\exp \left(\left(-\lambda_{1}+2 \nu-f\right) i \varphi+\left(-\lambda_{1}-2 e+f\right) i \theta\right) \tilde{W}(a(r)) \quad(n \in Z(N)) .
\end{aligned}
$$

Proof. We have $\tilde{W}(n g)=\eta(n) \tilde{W}(g)=\tilde{W}(g)$ for $g \in \tilde{G}$ and $n \in Z(N)=$ $[N, N]$, hence $\tilde{W}(h)=\tilde{W}\left(z(\varphi) a(r) k^{\prime}(\theta)\right)$. Write

$$
\begin{aligned}
z(\varphi) a(r) k^{\prime}(\theta) & =\exp \left(\frac{2}{3} i \varphi\right) a(r)\left(\begin{array}{lll}
e^{i \alpha} & \\
& e^{i \beta} & \\
& e^{-i(\alpha+\beta)}
\end{array}\right) ; \\
\alpha & =\frac{1}{3} \varphi+\theta, \beta=-\frac{2}{3} \varphi .
\end{aligned}
$$

We then have, by (5.7) and (6.3),

$$
\begin{aligned}
& \tilde{W}\left(z(\varphi) a(r) k^{\prime}(\theta)\right) \\
& =\exp \left(\left(-\lambda_{1}-\lambda_{2}+3 \nu\right) \frac{2}{3} i \varphi\right) \exp \left(-\left(\lambda_{1}+2 e-f\right) i \alpha-\left(\lambda_{2}+e-2 f\right) i \beta\right) \tilde{W}(a(r)) \\
& =\exp \left(\left(-\lambda_{1}+2 \nu-f\right) i \varphi+\left(-\lambda_{1}-2 e+f\right) i \theta\right) \tilde{W}(a(r)) .
\end{aligned}
$$

We now calculate $L^{\omega}(s, \tilde{W}, \Phi)$ for the above $\tilde{W}$ and for $\Phi=\Phi_{p}$ with $p=\left(m_{1}, n_{1}, m_{2}, n_{2}\right)$. By (6.2) (1) we assume that $n_{1}+n_{2}-m_{1}-m_{2}=\omega_{1}$. Then (6.2) (2) and (6.4), together with the Iwasawa decomposition $\tilde{H}=$ $Z(N) Z(\tilde{H}) A K^{\prime}$, imply that $c_{0}^{-1}$ times $L^{\omega}(s, \tilde{W}, \Phi)$ equals 


$$
\begin{aligned}
\int_{0}^{2 \pi} \exp \left(\left(-\lambda_{1}+2 \nu-f+n_{1}-m_{1}+n_{2}-m_{2}\right) i \varphi\right) d \varphi \\
\quad \times \int_{0}^{2 \pi} \exp \left(\left(-\lambda_{1}-2 e+f+n_{1}-m_{1}-n_{2}+m_{2}\right) i \theta\right) d \theta \\
\quad \times \Gamma\left(s+\omega_{0}-\frac{1}{2} \omega_{1}+n_{1}+n_{2}\right) \int_{0}^{\infty} W(a(r)) r^{2\left(s+\omega_{0}-1\right)} d r / r .
\end{aligned}
$$

Hence $L^{\omega}(s, \tilde{W}, \Phi)$ does not vanish only if

$$
-\lambda_{1}+2 \nu-f+n_{1}-m_{1}+n_{2}-m_{2}=-\lambda_{1}-2 e+f+n_{1}-m_{1}-n_{2}+m_{2}=0,
$$

namely,

$$
n_{1}-m_{1}=\lambda_{1}-\nu+e, n_{2}-m_{2}=-\nu-e+f
$$

Suppose that (6.5) is the case. Then, firstly, the above condition $n_{1}+n_{2}-m_{1}-m_{2}=\omega_{1}$ is equivalent to $f=-\lambda_{1}+2 \nu+\omega_{1}$. Secondly, the "greatest common divisor" of $\left\{\Gamma\left(s+\omega_{0}-\frac{1}{2} \omega_{1}+n_{1}+n_{2}\right)\right\}_{p}$ is written as $\Gamma\left(s+\omega_{0}+c(e)\right)$ with

$$
\begin{aligned}
c(e): & =-\frac{1}{2} \omega_{1}+\frac{1}{2}\left\{\left(\lambda_{1}-\nu+e\right)+\left|\lambda_{1}-\nu+e\right|+(-\nu-e+f)+|-\nu-e+f|\right\} \\
& =\frac{1}{2}\left\{\left|e+\lambda_{1}-\nu\right|+\left|e+\lambda_{1}-\nu-\omega_{1}\right|\right\} .
\end{aligned}
$$

Summing up, we have established

Proposition (6.7). Let $\tilde{W}$ be as in (6.3). Then $L^{\omega}\left(s, \tilde{W}, \Phi_{p}\right)$ does not vanish for some $p$ only if the following condition is satisfied:

$$
f=-\lambda_{1}+2 \nu+\omega_{1}
$$

If this is the case, the "greatest common divisor" of $\left\{L^{\omega}(s, \tilde{W}, \Phi)\right\}_{p}$ is written as

$$
\Gamma\left(s+\omega_{0}+c(e)\right) \int_{0}^{\infty} W(a(r)) r^{2\left(s+\omega_{0}-1\right)} d r / r
$$

where c(e) is defined by (6.6).

Let $L^{\omega}\left(s, \tilde{\pi}_{\lambda, \nu}^{*}\right)$ be the "greatest common divisor" of the family $\left\{L^{\omega}\left(s, \tilde{W}, \Phi_{p}\right)\right\}$, where $\tilde{W}$ runs through $K$-finite vectors in the Whittaker model $W h_{\eta}\left(\tilde{\pi}_{\lambda, \nu}^{*}\right)$ and $\Phi$ runs through the Schwartz space $\mathscr{S}(W)$. Combining the above proposition with (5.6) we can show the following 
Theorem (6.8). Let $\omega$ be the quasi-character of $\mathbf{C}^{\times}$fixed via (6.1) with two parameters $\omega_{0} \in \mathbf{C}$ and $\omega_{1} \in \mathbf{Z}$. Then $L^{\omega}\left(s, \tilde{\pi}_{\lambda, \nu}^{*}\right)$ is given as follows:

(1) If $\lambda_{2}-2 \nu \leq \omega_{1} \leq \lambda_{1}-2 \nu$, then $L^{\omega}\left(s, \tilde{\pi}_{\lambda, \nu}^{*}\right)$ coincides with

$$
\begin{aligned}
\left(\frac{\sqrt{b}}{2}\right)^{-s} & \Gamma\left(s+\omega_{0}-\frac{1}{2} \omega_{1}+\lambda_{1}-\nu\right) \\
& \times \Gamma\left(s+\omega_{0}+\frac{1}{2} \omega_{1}-\lambda_{2}+\nu\right) \Gamma\left(s+\omega_{0}+\frac{1}{2}\left(\left|\omega_{1}+\nu\right|+|\nu|\right)\right) .
\end{aligned}
$$

(2) If $\omega_{1} \leq \lambda_{2}-2 \nu$, then $L^{\omega}\left(s, \tilde{\pi}_{\lambda, \nu}^{*}\right)$ coincides with

$$
\begin{aligned}
\left(\frac{\sqrt{b}}{2}\right)^{-s} & \Gamma\left(s+\omega_{0}-\frac{1}{2} \omega_{1}+\lambda_{1}-\nu\right) \\
& \times \Gamma\left(s+\omega_{0}-\frac{1}{2} \omega_{1}-\nu\right) \Gamma\left(s+\omega_{0}+\frac{1}{2}\left(\left|\omega_{1}-\lambda_{2}+\nu\right|+\left|-\lambda_{2}+\nu\right|\right)\right) .
\end{aligned}
$$

(3) If $\omega_{1} \geq \lambda_{1}-2 \nu$, then $L^{\omega}\left(s, \tilde{\pi}_{\lambda, \nu}^{*}\right)$ coincides with

$$
\begin{aligned}
\left(\frac{\sqrt{b}}{2}\right)^{-s} & \Gamma\left(s+\omega_{0}+\frac{1}{2} \omega_{1}+\nu\right) \\
& \times \Gamma\left(s+\omega_{0}+\frac{1}{2} \omega_{1}-\lambda_{2}+\nu\right) \Gamma\left(s+\omega_{0}+\frac{1}{2}\left(i \omega_{1}-\lambda_{1}+\nu|+|-\lambda_{1}+\nu \mid\right)\right) .
\end{aligned}
$$

\subsection{Proof of Theorem (6.8)}

In this subsection, we prove the main theorem (6.8). By (6.7) our task is reduced to determine the "greatest common divisor" of

$$
\Gamma(s ; e, \mu):=\Gamma\left(s+\omega_{0}+c(e)\right) \int_{0}^{\infty} \tilde{W}(a(r)) r^{2\left(s+\omega_{0}-1\right)} d r / r
$$

for all $e \in \mathbf{Z}$ and $\mu \in \lambda+\mathbf{Z}_{\geq 0} \beta_{13}+\mathbf{Z}_{\geq 0} \beta_{32}$, where $\tilde{W}$ is a non-zero $T$-weight vector of weight $-\left(\lambda+e \beta_{13}+f \beta_{32}\right)$, contained in a $K$-type subspace $W h_{\eta}\left(\tilde{\pi}_{\lambda, \nu}^{*}\right)_{\mu} *$ of $W h_{\eta}\left(\tilde{\pi}_{\lambda, \nu}^{*}\right)$ $\left(W h_{\eta}\left(\tilde{\pi}_{\lambda, \nu}^{*}\right)_{\mu} *\right.$ is $K$-isomorphic to $\left.V_{\mu}^{*}\right)$. Here we may put $f=-\lambda_{1}+2 \nu+\omega_{1}, \mu=$ $\lambda+e \beta_{13}+f \beta_{32}+i \beta_{12}(i \geq 0)$. We then have

$$
\begin{gathered}
\Gamma(s ; e, \mu)=\Gamma\left(s+\omega_{0}+c(e)\right) M_{d_{\mu}-i}^{\mu}\left(2\left(s+\omega_{0}-1\right)\right) \\
d_{\mu}-i=d_{\lambda}+e+f+i=-\lambda_{2}+2 \nu+\omega_{1}+e+i .
\end{gathered}
$$

With an integer $e$ fixed, let $\Gamma(s ; e)$ be the "greatest common divisor" of the family $\{\Gamma(s ; e, \mu)\}_{\mu}$. 
Lemma (6.9). The above $\Gamma(s ; e)$ is given as follows.

(1) Suppose $f \leq 0$ and $e \geq-d_{\lambda}$ or $f \geq-d_{\lambda}$ and $e \leq 0$. Then

$$
\Gamma(s ; e)=\left\{\begin{array}{l}
\Gamma\left(s+\omega_{0}+c(e)\right) M_{d_{\lambda}+e}^{\lambda+(e-f) \beta_{13}}\left(2\left(s+\omega_{0}-1\right)\right) \cdots e \geq f ; \\
\Gamma\left(s+\omega_{0}+c(e)\right) M_{d_{\lambda}+f}^{\lambda+(f-e) \beta_{32}}\left(2\left(s+\omega_{0}-1\right)\right) \cdots e \leq f .
\end{array}\right.
$$

(2) Suppose $f \leq-d_{\lambda}$ and $e \leq-d_{\lambda}$. Then

$$
\Gamma(s ; e)=\Gamma\left(s+\omega_{0}+c(e)\right) M_{0}^{\lambda-\left(d_{\lambda}+f\right) \beta_{13}-\left(d_{\lambda}+e\right) \beta_{32}}\left(2\left(s+\omega_{0}-1\right)\right) .
$$

(3) Suppose $f \geq 0$ and $e \geq 0$. Then

$$
\Gamma(s ; e)=\Gamma\left(s+\omega_{0}+c(e)\right) M_{d_{\lambda}+e+f}^{\lambda+e \beta_{13}+f \beta_{32}}\left(2\left(s+\omega_{0}-1\right)\right)
$$

Proof. (1) follows from (5.6) (1) and (5.6) (2), (2) follows from (5.6) (3), and (3) follows from (5.6) (4).

We now determine the "greatest common divisor" of $\{\Gamma(s ; e)\}_{e}$, which coincides with the function $L^{\omega}\left(s, \tilde{\pi}_{\lambda, \nu}^{*}\right)$ in question. Note that the conditions $\lambda_{2}-2 \nu \leq \omega_{1} \leq \lambda_{1}-2 \nu, \omega_{1} \leq \lambda_{2}-2 \nu$ and $\omega_{1} \geq \lambda_{1}-2 \nu$ in (6.8) are equivalent with $-d_{\lambda} \leq f \leq 0, f \leq-d_{\lambda}$ and $f \geq 0$, respectively.

Proof of (6.8) (1). Suppose that $\lambda_{2}-2 \nu \leq \omega_{1} \leq \lambda_{1}-2 \nu$, namely, $-d_{\lambda} \leq f \leq 0$. In this case the desired expression for $L^{\omega}\left(s, \tilde{\pi}_{\lambda, \nu}^{*}\right)$ is nothing but $\Gamma(s ; f)$. In fact, one immediately checkes this by using (6.9) (1), the expression

$$
c(f)=\frac{1}{2}\left(\left|\omega_{1}+\nu\right|+|\nu|\right)
$$

(cf. (6.6)), and (5.2). Our task is, hence, to show that $\Gamma(s ; e) / \Gamma(s ; f)$ is a polynomial in $s$ for every $e \in \mathbf{Z}$. By (6.9) (1) and (5.6) (1), (2) we have

$$
\begin{aligned}
& \Gamma(s ; e) / \Gamma(s ; f)=\Gamma\left(s+\omega_{0}+c(e)\right) \Gamma\left(s+\omega_{0}+c(f)\right)^{-1} \\
& \times\left\{\begin{array}{l}
\Gamma\left(s+\omega_{0}-\frac{1}{2}\left(f+\lambda_{1}\right)\right) \Gamma\left(s+\omega_{0}-\frac{1}{2}\left(f+\lambda_{1}\right)-e+f\right)^{-1} \cdots e \geq f \\
\Gamma\left(s+\omega_{0}+\frac{1}{2}\left(f+\lambda_{1}\right)\right) \Gamma\left(s+\omega_{0}+\frac{1}{2}\left(f+\lambda_{1}\right)+e-f\right)^{-1} \cdots e \leq f
\end{array}\right.
\end{aligned}
$$

Hence $\Gamma(s ; e) / \Gamma(s ; f)$ is obviously a polynomial in $s$ if $c(e) \geq c(f)$. We therefore suppose that $c(e)<c(f)$. The definition (6.6) of $c(e)$ then implies in the case $e \geq f$ 


$$
\begin{aligned}
& f<\operatorname{Min}\left\{-\lambda_{1}+\nu,-\lambda_{1}+\nu+\omega_{1}\right\}, \\
& c(f)=-f-\lambda_{1}+\nu+\frac{1}{2} \omega_{1}=-\frac{1}{2}\left(f+\lambda_{1}\right), \\
& c(e) \geq c(f)-(e-f)=-\frac{1}{2}\left(f+\lambda_{1}\right)-e+f,
\end{aligned}
$$

and in the case $e \leq f$

$$
\begin{aligned}
& f>\operatorname{Max}\left\{-\lambda_{1}+\nu,-\lambda_{1}+\nu+\omega_{1}\right\}, \\
& c(f)=f+\lambda_{1}-\nu-\frac{1}{2} \omega_{1}=\frac{1}{2}\left(f+\lambda_{1}\right), \\
& c(e) \geq c(f)-(f-e)=\frac{1}{2}\left(f+\lambda_{1}\right)+e-f .
\end{aligned}
$$

These facts, together with above expression for $\Gamma(s ; e) / \Gamma(s ; f)$, show that $\Gamma(s ; e) / \Gamma(s ; f)$ is a polynomial in $s$ even if $c(e)<c(f)$. This completes the proof.

Proof of (6.8) (2). Suppose that $\omega_{1} \leq \lambda_{2}-2 \nu$, namely, $f \leq-d_{\lambda}$. In this case the desired expression for $L^{\omega}\left(s, \tilde{\pi}_{\lambda, \nu}^{*}\right)$ is nothing but $\Gamma\left(s ;-d_{\lambda}\right)$; one immediately checks this by using (6.9)(1) (or (2)), the expression $c\left(-d_{\lambda}\right)=\frac{1}{2}\left(\left|\omega_{1}-\lambda_{2}+\nu\right|+\right.$ $\left|-\lambda_{2}+\nu\right|$ ) and (5.6)(1) (or (3)). We shall show that $\Gamma(s ; e) / \Gamma\left(s ;-d_{\lambda}\right)$ is a polynomial in $s$ for every $e \in \mathbf{Z}$.

In the case $e \geq-d_{\lambda}$ we use (6.9) (1) and (5.6) (1) to get

$$
\begin{aligned}
\Gamma(s ; e) / \Gamma\left(s ;-d_{\lambda}\right)= & \Gamma\left(s+\omega_{0}+c(e)\right) \Gamma\left(s+\omega_{0}+c\left(-d_{\lambda}\right)\right)^{-1} \\
& \times \Gamma\left(s+\omega_{0}+\frac{1}{2}\left(f-\lambda_{1}\right)+d_{\lambda}\right) \Gamma\left(s+\omega_{0}+\frac{1}{2}\left(f-\lambda_{1}\right)-e\right)^{-1},
\end{aligned}
$$

and in the case $e \leq-d_{\lambda}$ we use (6.9) (2) and (5.6) (3) to get

$$
\begin{aligned}
\Gamma(s ; e) / \Gamma\left(s ;-d_{\lambda}\right) & =\Gamma\left(s+\omega_{0}+c(e)\right) \Gamma\left(s+\omega_{0}+c\left(-d_{\lambda}\right)\right)^{-1} \\
& \times \Gamma\left(s+\omega_{0}-\frac{1}{2}\left(f+\lambda_{1}\right)+\lambda_{2}\right) \Gamma\left(s+\omega_{0}-\frac{1}{2}\left(f+\lambda_{1}\right)+\lambda_{2}+d_{\lambda}+e\right)^{-1} .
\end{aligned}
$$

In both cases we immediately have $\Gamma(s ; e) / \Gamma\left(s ;-d_{\lambda}\right) \in \mathbf{C}[s]$ if $c(e) \geq c\left(-d_{\lambda}\right)$. Suppose, then, $c(e)<c\left(-d_{\lambda}\right)$. By (6.6) we see that if $e \geq-d_{\lambda}$ 


$$
\begin{aligned}
& -d_{\lambda}<\operatorname{Min}\left\{-\lambda_{1}+\nu,-\lambda_{1}+\nu+\omega_{1}\right\}, \\
& c\left(-d_{\lambda}\right)=d_{\lambda}-\lambda_{1}+\nu+\frac{1}{2} \omega_{1}=\frac{1}{2}\left(f-\lambda_{1}\right)+d_{\lambda}, \\
& c(e) \geq c\left(-d_{\lambda}\right)-\left(e+d_{\lambda}\right)=\frac{1}{2}\left(f-\lambda_{1}\right)-e,
\end{aligned}
$$

and that if $e \leq-d_{\lambda}$

$$
\begin{aligned}
& -d_{\lambda}>\operatorname{Max}\left\{-\lambda_{1}+\nu,-\lambda_{1}+\nu+\omega_{1}\right\}, \\
& c\left(-d_{\lambda}\right)=-d_{\lambda}+\lambda_{1}-\nu-\frac{1}{2} \omega_{1}=-\frac{1}{2}\left(f+\lambda_{1}\right)+\lambda_{2}, \\
& c(e) \geq c\left(-d_{\lambda}\right)+\left(e+d_{\lambda}\right)=-\frac{1}{2}\left(f+\lambda_{1}\right)+\lambda_{2}+d_{\lambda}+e .
\end{aligned}
$$

We hence have $\Gamma(s ; e) / \Gamma\left(s ;-d_{\lambda}\right) \in \mathbf{C}[s]$ in both cases. This completes the proof.

Proof of (6.8) (3). Suppose that $\omega_{1} \geq \lambda_{1}-2 \nu$, namely, $f \geq 0$.. In this case the desired expression for $L^{\omega}\left(s, \tilde{\pi}_{\lambda, \nu}^{*}\right)$ is nothing but $\Gamma(s ; 0)$; one immediately checks this by using (6.9) (1) (or (3)), the expression $c(0)=\frac{1}{2}\left(\left|\omega_{1}-\lambda_{1}+\nu\right|+\right.$ $\left|-\lambda_{1}+\nu\right|$ ), and (5.6) (2) (or (4)). We shall show that $\Gamma(s ; e) / \Gamma(s ; 0)$ is a polynomial in $s$ for every $e \in \mathbf{Z}$.

In the case $e \geq 0$ we use (6.9) (3) and (5.6) (4) to get

$$
\begin{aligned}
\Gamma(s ; e) / \Gamma(s ; 0)= & \Gamma\left(s+\omega_{0}+c(e)\right) \Gamma\left(s+\omega_{0}+c(0)\right)^{-1} \\
& \times \Gamma\left(s+\omega_{0}+\frac{1}{2}\left(f-\lambda_{1}\right)\right) \Gamma\left(s+\omega_{0}+\frac{1}{2}\left(f-\lambda_{1}\right)-e\right)^{-1},
\end{aligned}
$$

and in the case $e \leq 0$ we use (6.9) (1) and (5.6) (2) to get

$$
\begin{aligned}
\Gamma(s ; e) / \Gamma(s ; 0)= & \Gamma\left(s+\omega_{0}+c(e)\right) \Gamma\left(s+\omega_{0}+c(0)\right)^{-1} \\
& \times \Gamma\left(s+\omega_{0}-\frac{1}{2}\left(f-\lambda_{1}\right)\right) \Gamma\left(s+\omega_{0}-\frac{1}{2}\left(f-\lambda_{1}\right)+e\right)^{-1} .
\end{aligned}
$$

In both cases we immediately have $\Gamma(s ; e) / \Gamma(s ; 0) \in \mathbf{C}[s]$ if $c(e) \geq c(0)$. Suppose, then, $c(e)<c(0)$. By (6.6) we see that if $e \geq 0$ 


$$
\begin{gathered}
0<\operatorname{Min}\left\{-\lambda_{1}+\nu,-\lambda_{1}+\nu+\omega_{1}\right\}, \\
c(0)=-\lambda_{1}+\nu+\frac{1}{2} \omega_{1}=\frac{1}{2}\left(f-\lambda_{1}\right), \\
c(e) \geq c(0)-e=\frac{1}{2}\left(f-\lambda_{1}\right)-e,
\end{gathered}
$$

and that if $e \leq 0$

$$
\begin{gathered}
0>\operatorname{Max}\left\{-\lambda_{1}+\nu,-\lambda_{1}+\nu+\omega_{1}\right\}, \\
c(0)=\lambda_{1}-\nu-\frac{1}{2} \omega_{1}=-\frac{1}{2}\left(f-\lambda_{1}\right), \\
c(e) \geq c(0)+e=-\frac{1}{2}\left(f-\lambda_{1}\right)+e .
\end{gathered}
$$

We hence have $\Gamma(s ; e) / \Gamma(s ; 0) \in \mathbf{C}[s]$ in both cases. This completes the proof of Theorem (6.8).

\section{$\S 7$. Comments for the Case of Principal Series Representations}

The method of computation for local zeta integral applies not only for the discrete series but also for the principal series. In fact the case of the principal series is even simpler than the case of the discrete series. We remark some basic facts for the case of the principal series in this section.

\subsection{Definition of the principal series}

Let us recall the definition of the principal series representations. The centralizer $M$ of $a$ in $K$ is given by

$$
M=\left\{\left(\begin{array}{lll}
e^{i \theta} & & \\
& e^{-2 i \theta} & \\
& & e^{i \theta}
\end{array}\right) \mid \theta \in \mathbf{R}\right\} .
$$

For each integer $n \in \mathbf{Z}$, we denote by $\chi_{n}$ the character of $M$ defined by

$$
\operatorname{diag}\left(e^{i \theta}, e^{-2 i \theta}, e^{i \theta}\right) \in M \mapsto e^{i n \theta} \in \mathbf{C}^{*}
$$

Let $\nu \in \mathfrak{a}_{\mathbf{c}}^{*}=\operatorname{Hom}_{\mathbf{R}}(\mathfrak{a}, \mathbf{C})$ be a complex-valued linear form on $\mathfrak{a}$, and $\rho$ the half-sum of the positive roots in $\Phi(\mathfrak{g}, \mathfrak{a})$. Let $\xi_{\nu+\rho}: A \rightarrow \mathrm{C}^{*}$ be a continuous character of $A$ given by

$$
a \in A \mapsto e^{(\nu+\rho)(\log a)} \in \mathbf{C}^{*} .
$$


Then we can define a character $\chi_{n, \nu+\rho}$ of a parabolic subgroup $P=M A N$ by

$$
\chi_{n, \nu+\rho}(a)=\chi_{n}(m) \xi_{\nu+\rho}(a) \text { for } p=\operatorname{man} \in P(m \in M, a \in A, n \in N) .
$$

The representation space of $\operatorname{Ind}_{P}^{G}\left(\chi_{n, \nu+\rho}\right)$ is given by

$$
L_{n}^{2}(K)=\left\{f \in L^{2}(K) \mid f(m k)=\chi_{n}(m) f(k) m \in M, k \in K\right\}
$$

Any function $f$ is extended to a function $\tilde{f}$ on $G$ via a decomposition $g=p k$ $\in G=P K$ :

$$
\tilde{f}(g)=\chi_{n, \nu+\rho}(p) f(k) .
$$

Since $M=P \cap K$ and $f \in L_{n}^{2}(K)$, this is well-defined. The action $\pi$ of $G$ on $H=\left\{\tilde{f} \mid f \in L_{n}^{2}(K)\right\}$ is defined by the right regular action

$$
\pi(g) \tilde{f}(x)=\tilde{f}(x g)
$$

Then $\pi=\operatorname{Ind}_{P}^{G}\left(\chi_{n, \nu+\rho}\right)$ is a Hilbert representation.

\subsection{K-types}

We want to describe the $K$-types of the principal series. It is enough to know the action of $K$ on $L_{n}^{2}(K)$. The multiplicity of $\tau$ of $\hat{K}$ in $\operatorname{Ind}_{P}^{G}\left(\chi_{n, \nu+\rho}\right)$ is given by

$$
[\pi, \tau]=\sum_{\omega \in K \widehat{\cap M}}[\tau: \omega] \cdot\left[\chi_{n}: \omega\right],
$$

where $[\tau: \omega]$ and $\left[\chi_{n}: \omega\right]$ are the multiplicities of $\omega$ in $\left.\tau\right|_{K \cap M}$ and $\left.\chi_{m}\right|_{K \cap M}$ respectively (cf. Knapp $[\mathrm{K}]$, or Vogan $\left[\mathrm{V},\left(4.5 .^{*}\right)\right]$ ). In our case, $K \cap M=M$. Hence

$$
[\pi: \tau]=\left[\left.\tau\right|_{M}: \chi_{n}\right]
$$

Let $\tau=\tau_{\mu}$ be an element of $\hat{K}$ with highest weight $\mu=\left(\mu_{1}, \mu_{2}\right)$. Then the weights of $\tau$ are given by

$$
\mu, \mu-\beta_{12}, \mu-2 \beta_{12}, \cdots, \mu-d_{\mu} \beta_{12}=\left(\mu_{2}, \mu_{1}\right),
$$

where $d_{\mu}=\mu_{1}-\mu_{2}$. The restriction of each weight space with weight $\mu-k \beta_{12}$ to $M$ is an $M$-module of dimension 1 with respect to a character

$$
\chi_{\left\{\mu_{1}-k\right\}-2\left\{\mu_{2}+k\right\}}=\chi_{\left\{\left(\mu_{1}-2 \mu_{2}\right)-3 k\right\}} .
$$

Therefore 


$$
\left[\pi: \tau_{\mu}\right]=\#\left\{k \in \mathbf{Z} \mid 0 \leq k \leq \mu_{1}-\mu_{2},\left(\mu_{1}-2 \mu_{2}\right)-3 k=n\right\} \leq 1 .
$$

In particular when $\mu_{1}=\mu_{2},\left[\pi: \tau_{\mu}\right]=1$ if and only if $\left(\mu_{1}, \mu_{2}\right)=(-n,-n)$. Now it is easy to check the following.

Lemma (7.2). The multiplicity of $\tau_{\mu}$ in $\operatorname{Ind}_{P}^{G}\left(\chi_{n, \nu+\rho}\right)$ is 1 if and only if

$$
\mu=m_{1} \beta_{13}+m_{2} \beta_{32}+(-n,-n) \text { with some non-negative integers } m_{1}, m_{2}
$$

Otherwise it is 0.

\subsection{Infinitesimal character}

We calculate the eigenvalues of the Casimir operator on the principal series representations in this subsection. In general, given a basis $\left\{X_{i}\right\}_{1 \leq i \leq 8}$ of $\mathrm{g}$, let $\left\{g^{i j}\right\}_{1 \leq i, j \leq 8}$ be the inverse of an invertible matrix $\left\{\operatorname{tr}\left(X_{i} X_{j}\right)\right\}_{1 \leq i, j \leq 8}$. Then an element

$$
L=\sum_{i, j=1}^{8} g^{i j} X_{i} X_{j}
$$

of the universal envelopping algebra $U(\mathfrak{g})$ is a constant multiple of the Casimir operator, because a bilinear form defined by $(X, Y) \in \mathfrak{g} \times \mathfrak{g} \rightarrow \operatorname{tr}(X Y)$ is a non-zero constant multiple of the Killing form. Then element $L$ does not depend on the choice of basis $\left\{X_{i}\right\}$.

The Cartan involution on $\mathfrak{g}$ is given by

$$
\theta(X)=-{ }^{t} \bar{X} \quad \text { for } X \in g .
$$

Put $\mathfrak{n}_{-}=\theta(\mathfrak{n})$. Then it is the nilpotent algebra opposite to $\mathfrak{n}$. Let $\mathfrak{m}$ be the Lie algebra of $M$ or the centralizer of $\mathfrak{n}$ in $\mathfrak{l}$. Then we have a decomposition:

$$
\mathfrak{g}=\mathfrak{n}_{-} \oplus \mathfrak{a} \oplus \mathfrak{m} \oplus \mathfrak{n}
$$

The expression of the operator $L$ with respect to a basis

$$
\left\{\theta\left(E_{1}\right), \theta\left(E_{2,+}\right), \theta\left(E_{2,-}\right), H_{1}, M_{1}=\sqrt{-1} \operatorname{diag}(1,-2,1), E_{1}, E_{2 .^{+}}, E_{2,-}\right\}
$$

is given by 


$$
\begin{aligned}
L= & \frac{1}{2} H_{1}^{2}-\frac{1}{6} M_{1}^{2} \\
& -\frac{1}{4}\left\{E_{1} \theta\left(E_{1}\right)+\theta\left(E_{1}\right) E_{1}+E_{2,+} \theta\left(E_{2,+}\right)+\theta\left(E_{2,+}\right) E_{2,+}+E_{2,-} \theta\left(E_{2,-}\right)+\theta\left(E_{2,-}\right) E_{2,-}\right\} \\
= & \frac{1}{2} H_{1}^{2}-\frac{1}{6} M_{1}^{2}-\frac{1}{2}\left\{\theta\left(E_{1}\right) E_{1}+\theta\left(E_{2,+}\right) E_{2,+}+\theta\left(E_{2,-}\right) E_{2,-}\right\}+2 H_{1} .
\end{aligned}
$$

The $C^{\infty}$-vectors $H_{\infty}$ of the representation space $H$ of the principal series representation $\pi$ is contained in $C^{\infty}(G)$, the space of $C^{\infty}$-functions on $G$. Since $L$ is in the center of $U(\mathfrak{g})$, it is both left and right invariant tensor field. Therefore on $C^{\infty}(G)$ the right regular action of $L$ coincides with the left regular one. The left regular action of $H_{1}, M_{1}, E_{1}, E_{2, \pm}$ on $\tilde{f} \in H_{\infty}$ are given by

$$
H_{1} \tilde{f}=-(\nu+\rho) \tilde{f}, \quad M_{1} \tilde{f}=\sqrt{-1} n \tilde{f}, \quad E_{1} \tilde{f}=E_{2, \pm} \tilde{f}=0 .
$$

Therefore

$$
L \tilde{f}=\left\{\frac{1}{2}(-\nu-\rho)^{2}+\frac{1}{6} n^{2}-2(\nu+\rho)\right\} \tilde{f}=\left(\frac{1}{2} \nu^{2}+\frac{1}{6} n^{2}-2\right) \tilde{f} .
$$

Here we identify $\mathfrak{a}_{\mathrm{c}}^{*}$ with $\mathbf{C}$ by mapping $e_{1} \in \mathfrak{a}_{\mathrm{c}}^{*}$ to $1 \in \mathbf{C}$. This is the infinitesimal chracter of the principal series representation.

\subsection{Whittaker functions of the principal series}

Let $\pi^{*}$ be the contragradient representation to $\pi=\pi_{n, \nu}$. Then

$$
\pi^{*}=\pi_{-n,-\nu}=\operatorname{Ind}_{P}^{G}\left(1_{N} \otimes \xi_{-\nu+\rho} \otimes \chi_{-n}\right) .
$$

The $K$-types of $\pi^{*}$ is given by

$$
\left.\pi^{*}\right|_{K}=\otimes_{m_{1}, m_{2} \geq 0} \tau_{(n, n)+m_{1} \beta_{13}+m_{2} \beta_{32}} .
$$

The eigenvalue of the Casimir operator is the same as $\pi$ :

$$
\Omega f=\left(\frac{1}{2} \nu^{2}+\frac{1}{6} n^{2}-2\right) f ; \quad f \text { is a smooth vector of } \pi^{*} .
$$

Fix an intertwining operator $\iota \in \operatorname{Hom}_{(\mathrm{g}, K)}\left(\pi^{*}, C_{\eta}^{\infty}(N \backslash G)\right)$. Then for each $K$ type $\left(\tau^{*}, V^{*}\right)$ which occurs with multiplicity one in $\pi^{*}$, the restriction $\iota$ gives an element $F=F_{\iota}$ of $C_{\eta, \tau}^{\infty}(N \backslash G / K)$ via correspondence

$$
\begin{gathered}
\iota \mid V^{*} \in \operatorname{Hom}_{K}\left(V^{*}, C_{\eta}^{\infty}(N \backslash G)\right) \cong C_{\eta, \tau}^{\infty}(N \backslash G / K) . \\
\left(v^{*} \rightarrow\left(g \rightarrow<v^{*}, F(g)>\right)\right) \leftrightarrow F .
\end{gathered}
$$



function

Since the eigenvalue of the Casimir operator $\Omega$ for $\pi^{*}$ is $\frac{1}{2} \nu^{2}+\frac{1}{6} n^{2}-2$, the

$$
g \in G \rightarrow<v^{*}, F(g)>\quad \text { on } G
$$

satisfies a differential equation

$$
\Omega<v^{*}, F(g)>=\left(\nu^{2} / 2+n^{2} / 6-2\right)<v^{*}, F(g)>.
$$

Let $\left(\tau^{*}, V^{*}\right)=\left(\tau_{(n, n)}, V_{(n, n)}\right)$, i.e. 1-dimensional representation of $K$ with highest weight $(n, n)$. Fix a generator $v_{0}$ in $V^{*}$, and write $F(g)=c_{0}(g) v_{0}$ with $c_{0}(g) \in C^{\infty}(G)$. Then the radial part of $c_{0}(g)$ satisfies

$$
\left[\frac{d^{2}}{d r^{2}}-\frac{3}{r} \frac{d}{d r}+\eta_{+} \eta_{-}+\frac{1}{r^{2}}\left(4-\nu^{2}\right)\right] c_{0}(a)=0 .
$$

Now assume that the functions in the image of the intertwining operator $\iota$ is slowly increasing at infinity on the split component $A$. Then up to scalar multiple

$$
c_{0}(a)=r^{3 / 2} W_{0, \nu}(2 \sqrt{b} r) \quad\left(b=-\eta_{+} \eta_{-}>0\right) .
$$

This result coincides with the construction of Goodman-Wallach (cf. [G-W], Lemma 7.3, (II)).

The computation in $\S 5$ for the discrete series goes through also for the principal series by setting

$$
d_{\lambda}=k=0 \quad \text { and } \quad \lambda_{1}=\lambda_{2}=\nu
$$

\section{$\S 8$. Comments on $L$-function and its Special Values}

As far as the authors know, the only complete result and proof for the functional equation of $L$-function associated with the "standard representation" of the dual group $G L(3, \mathrm{C})$ are those of Murase and Sugano [M-S], who discuss these for holomorphic modular forms.

The method indicated by Gelbart and Piatetski-Shapiro [G-Ps] does not seem to be worked out completely up to now. Because we have done the local computation at the real place, the other local problem is to work at $p$-adic ramified places, among others, at the place dividing the discriminant of the given Hermitian forms defining $U(2,1)$.

The results of [M-S] give not only the functional equation but also a description of certain special values of $L$-functions in terms of Petersson inner metric. Consider the special case when the weight of holomorphic modular forms is 3 . The interesting thing is that, contrary to the case of Hilbert modular surfaces (cf. [O2]), the special values are not the periods of 2 -forms on algebraic surfaces, but 
a product of such periods because of the Riemann-Hodge period relation for Picard modular surfaces.

This is a fact. But we wonder why it is, and what is the meaning of this !

Recently Kenji Taniguchi obtained explicit formulae for the discrete series Whittaker functions with minimal $K$-types for certain groups of rank 1 (cf. [T]). It seems interesting to have functional equation of $L$-functions on $S p(1,1)$ by the method of Andrianov.

\section{References}

[G-Ps] Gelbert, S. and Piatetski-Shapiro, I., Automorphic forms and $L$-functions for the unitary group, in Lie Group Representations II, Lecture Notes in Math., 1041, SpringerVerlag, New York, (1984), 141-184.

[G-W] Goodman, R. and Wallach, N., Whittaker vectors and conical vectors, J. Funct. Anal., 39 (1980), 199-279.

[H-S] Hecht, H. and Schmid, W., A proof of Blattner's conjecture, Invent. Math. 31 (1975), $129-154$.

[K] Kostant, B., On Whittaker Vectors and Representation Theory, Invent. Math., 48 (1978), $101-184$.

[MOS] Magnus, W., Oberhettinger, F. and Soni, R. P., Formulas and Theorems for the Special Functions of Mathematical Physics, 3rd ed. Springer-Verlag, 1966.

[M-S] Murase, A. and Sugano, T., $L$-functions of holomorphic cusp forms on $U(2,1)$, Preprint.

[O] Oda, T., An explicit integral representation of Whittaker functions on $\mathrm{Sp}(2 ; \mathbf{R})$ for the large discrete series representations, To appear in Tohoku J. Math.

[O2] - Periods of Hilbert modular surfaces, Birkhäuser, 1982.

[R-S] Reed, M. and Simon, B., Methods of modern mathematical physics I: Functional Analysis, Academic Press, 1980.

[Sh] Shalika, J.A., The multiplicity one theorem for $G L_{n}$, Ann. of Math., 100 (1974), $171-$ 193.

[S] Schmid, W., On the realization of the discrete series of a semisimple Lie group, Rice Univ. Studies, 56 (1970), 99-108.

[V] Vogan, D. Jr., Gelfand-Kirillov Dimension for Harish-Chandra Modules, Invent. Math. 49 (1978), 75-98.

[W] Wallach, N., Asymptotic expansions of generalized matrix entries of representations of real reductive groups, Lie Group Representations I, Lecture Notes in Math., 1024 (1984), $287-369$.

[W-W] Whittaker, E. T. and Watson, G. N., A course of modern analysis, Cambridge Univ. Press, 4ed., 1965.

[Y-I] Yamashita, H., Embedding of discrete series into induced representations of semisimple Lie groups, I, General theory and the case of SU(2, 2), Japan J. Math., 16 (1990), 31-95.

[Y-II ] - Embedding of discrete series into induced representations of semi-simple Lie groups, II, -Generalized Whittaker models for $S U(2,2)$-, J. Math. Kyoto Univ., 31-1 (1991), 543-571.

[Y-III $] \longrightarrow$, Associated varieties and Gelfand-Kirillov dimension for the discrete series of a semi-simple Lie group, Preprint.

[T] Taniguchi, K., Discrete series Whittaker functions of $S U(n, 1)$ and $\operatorname{Spin}(2 n, 1)$, Preprint UTMS95-23. 
